\title{
What is a Sobolev space for the Laguerre function systems?
}

by

\author{
B. Bongionnni (Santa Fe) and J. L. Torrea (Madrid)
}

\begin{abstract}
We discuss the concept of Sobolev space associated to the Laguerre operator $L_{\alpha}=-y \frac{d^{2}}{d y^{2}}-\frac{d}{d y}+\frac{y}{4}+\frac{\alpha^{2}}{4 y}, y \in(0, \infty)$. We show that the natural definition does not agree with the concept of potential space defined via the potentials $\left(L_{\alpha}\right)^{-s}$. An appropriate Laguerre-Sobolev space is defined in order to achieve that coincidence. An application is given to the almost everywhere convergence of solutions of the Schrödinger equation. Other Laguerre operators are also considered.
\end{abstract}

1. Introduction. We start with a naive description of our aim in writing this paper. Let $\mathbf{L}$ be a linear second order differential operator, selfadjoint with respect to a certain measure $\mu$. Different techniques (see for example (2)) allow us to define the "Riesz potentials" $\mathbf{L}^{-s}, s>0$. Therefore, we can consider the "potential space" $\mathbf{L}_{s}^{p}, 1<p<\infty$, as $\mathbf{L}^{-s / 2}\left(L^{p}(\mu)\right)$, the collection of functions $f$ such that there exists $g \in L^{p}(\mu)$ with $f=\mathbf{L}^{-s / 2}(g)$.

In general, the second order operator $\mathbf{L}$ admits a certain factorization $\mathbf{L}=\sum_{i} \mathcal{D}_{i}^{*} \mathcal{D}_{i}$, where the $\mathcal{D}_{i}$ are first order differential operators with adjoints $\mathcal{D}_{i}^{*}$ (with respect to $\mu$ ). Then it is also usual to define the "Riesz transforms" $R_{i}=\mathcal{D}_{i} \circ \mathbf{L}^{-1 / 2}$ and analyze their boundedness on $L^{p}(\mu)$ (see [16], [20]). Several motivations can be given for the study of these Riesz transforms. For example, the boundedness in $L^{p}$ of operators like $\mathcal{D}_{i}^{2} \circ \mathbf{L}^{-1}$ (usually called Riesz transforms of second order) leads rather easily to "a priori" estimates in $L^{p}$ for the equation $\mathbf{L} u=f$ : just observe that $\left\|\mathcal{D}_{i}^{2} \circ \mathbf{L}^{-1} g\right\|_{p} \leq C\|g\|_{p}$ can be written in this case as $\left\|\mathcal{D}_{i}^{2} u\right\|_{p} \leq C\|f\|_{p}$.

A second motivation (in fact the motivation of this note) is the following. Given a natural number $k$, let us define the (Sobolev) space $W_{p}^{k}$ as the collection of functions in $L^{p}$ such that the $k$ th derivatives $\mathcal{D}_{i}^{k} f$ belong to $L^{p}$. Suppose that the Riesz transforms of order $k, \mathcal{D}_{i}^{k} \circ \mathbf{L}^{-k / 2}$, satisfy $\left\|\mathcal{D}_{i}^{k} \circ \mathbf{L}^{-k / 2} f\right\|_{p} \sim\|f\|_{p}$. This last equivalence could be written (at least formally) as $\left\|\mathcal{D}_{i}^{k} f\right\|_{p} \sim\left\|\mathbf{L}^{k / 2} f\right\|_{p}$. In other words, the spaces $W_{p}^{k}$ and $\mathbf{L}_{k}^{p}$

2000 Mathematics Subject Classification: Primary 42B35; Secondary 42C05.

Key words and phrases: Laguerre functions, Sobolev spaces, Riesz transforms. 
would coincide. As the spaces $\mathbf{L}_{s}^{p}$ have a meaning for all $s>0$ (even noninteger), one could say that the potential space $\mathbf{L}_{s}^{p}$ is the space of functions in $L^{p}$ whose "sth derivative" is in $L^{p}$. We observe that if $\varphi_{n}$ is an eigenfunction of $\mathbf{L}$ with eigenvalue $\lambda_{n} \neq 0$ then $\mathbf{L}^{-s} \varphi_{n}=\lambda_{n}^{-s} \varphi_{n}$. Hence the last interpretation of the sth derivative is particularly simple to understand.

If the above ideas are directly applied to the Laguerre differential operator (for Laguerre functions), one finds something that can be considered a surprise. The expected definition of Sobolev space of order $k$, that is, the set of functions in $L^{p}$ such that the derivatives (according to the natural factorization of the differential operator) of order $k$ belong to $L^{p}$, does not match the definition of potential spaces (see Definitions 2-4 and Theorems 3 and 2). The main purpose of the paper is to clarify and make precise what could be the most appropriate definition of Sobolev spaces for the Laguerre operator. Our work was inspired by [21] and [13].

It is a common fact that if a concept is developed for Laguerre functions then the analogous concept can be developed in an easier way for Hermite functions. That happens in this work and so we devote Section 2 to Hermite functions. A comment about the dimension is in order here. The motivation of this paper is essentially one-dimensional, but in the case of Hermite functions, the theory has no added difficulty in several variables, so we present our results for the Hermite operator in that context.

The knowledge of a sharp enough power weighted theory for a Laguerre function system can be transferred to another Laguerre function system (see [1]). That is why we develop a weighted theory of Sobolev and potential spaces for a particular system of Laguerre functions and then we transfer it in an easy way to other systems (see Section 5).

Finally, in the last section we present a simple application to the pointwise convergence of solutions of the Schrödinger equation.

We discuss briefly the case of the Hermite operator

$$
H=-\Delta+|x|^{2}, \quad x \in \mathbb{R}^{d} .
$$

$H$ is self-adjoint on the set $C_{c}^{\infty}(\mathbb{R})$ of infinitely differentiable functions with compact support. The underlying measure will be the Lebesgue measure.

For each $s>0$, the Hermite potential, $H^{-s}$, is defined for $f \in L^{2}(\mathbb{R}, d x)$, by the formula

$$
H^{-s} f(x)=\frac{1}{\Gamma(s)} \int_{0}^{\infty} e^{-t H} f(x) t^{s} \frac{d t}{t}, \quad x \in \mathbb{R}^{d},
$$

where $\left\{e^{-t H}\right\}_{t \geq 0}$ is the heat semigroup associated to $H$. The corresponding potential spaces, $\mathcal{L}_{s}^{p}(w)=H^{-s / 2}\left(L^{p}(w)\right)$, are defined in (6) with respect to an absolutely continuous measure $w(x) d x, w$ being a weight in $A_{p}$. For the reader's convenience we recall that a positive function $w$ is said to belong 
to the Muckenhoupt class $A_{p}, 1<p<\infty$, if the Hardy-Littlewood maximal operator is bounded from $L^{p}(w(x) d x)$ into $L^{p}(w(x) d x)$, and $w$ is said to belong to the class $A_{1}$ if the Hardy-Littlewood maximal operator is bounded from $L^{1}(w(x) d x)$ into weak- $L^{1}(w(x) d x)$.

The operator $H$ can be factorized as $H=\frac{1}{2} \sum_{j=1}^{d}\left(A_{j} A_{-j}+A_{-j} A_{j}\right.$ ) (see (7)), where $A_{j}$ and $A_{-j}$ are first order differential operators.

Definition 1. Given $k \in \mathbb{N}$, the Hermite-Sobolev space of order $k$, denoted by $W^{k, p}(w)$, is the set of functions $f \in L^{p}(w)$ such that

$$
\overbrace{A_{j} \cdots A_{j}}^{m \text { times }} f=A_{j}^{m} f \in L^{p}(w), \quad 1 \leq m \leq k, 1 \leq j \leq d,
$$

with the norm

$$
\|f\|_{W^{k, p}(w)}=\sum_{j=1}^{d} \sum_{1 \leq m \leq k}\left\|A_{j}^{m} f\right\|_{L^{p}(w)}+\|f\|_{L^{p}(w)} .
$$

The following theorem will be proved in Section 2 .

Theorem 1. Let $k \in \mathbb{N}, 1<p<\infty$, and $w \in A_{p}$. Then

$$
W^{k, p}(w)=\mathfrak{L}_{k}^{p}(w)
$$

and the norms $\|\cdot\|_{W^{k, p}(w)}$ and $\|\cdot\|_{\mathfrak{L}_{k}^{p}(w)}$ are equivalent.

Of course, to prove this theorem, we shall need a boundedness result for higher order Riesz transforms (see Theorem 5).

Regarding the Laguerre operator

$$
L_{\alpha}=-y \frac{d^{2}}{d y^{2}}-\frac{d}{d y}+\frac{y}{4}+\frac{\alpha^{2}}{4 y}, \quad y \in(0, \infty),
$$

selfadjoint in the set $C_{c}(0, \infty)$, there is a natural domain of power weights $y^{\delta}$ for the boundedness on $L^{p}\left((0, \infty), y^{\delta} d y\right)$ of classical operators associated to $L_{\alpha}$ (see [1]), namely for $\alpha>-1,1<p<\infty$ and $\delta \in \mathbb{R}$,

$$
\left(\mathrm{C}_{\alpha}\right) \quad-\frac{\alpha}{2} p-1<\delta<p-1+\frac{\alpha}{2} p .
$$

In a parallel way to the Hermite case, we can define appropriate potential spaces for Laguerre functions.

Definition 2. Given $\alpha>-1,1<p<\infty, s>0$ and $\delta \in \mathbb{R}$ we define

$$
\mathfrak{W}_{\alpha, s}^{p}\left(y^{\delta}\right)=\left(L_{\alpha}\right)^{-s / 2}\left[L^{p}\left((0, \infty), y^{\delta} d y\right)\right]
$$

with the norm $\|f\|_{\mathfrak{W}_{\alpha, s}^{p}\left(y^{\delta}\right)}=\|g\|_{p, \delta}$, where $\left(L_{\alpha}\right)^{-s / 2} g=f$.

On the other hand, the Laguerre operator can be factorized as $L_{\alpha}=$ $\left(\delta^{\alpha}\right)^{*} \delta^{\alpha}+(\alpha+1) / 2$ (see (11)). Following the ideas we developed for the Hermite case, one can give the following. 
Definition 3. We denote by $\mathcal{W}_{\alpha}^{k, p}\left(y^{\delta}\right)$ the set of functions $f$ in $L^{p}\left((0, \infty), y^{\delta} d y\right)$ such that $\left(\delta^{\alpha}\right)^{m} f \in L^{p}\left((0, \infty), y^{\delta} d y\right), 0 \leq m \leq k$, with the norm

$$
\|f\|_{\mathcal{W}_{\alpha}^{k, p}\left(y^{\delta}\right)}=\sum_{m=0}^{k}\left\|\left(\delta^{\alpha}\right)^{m} f\right\|_{L^{p}\left((0, \infty), y^{\delta} d y\right)} .
$$

However, even though we shall prove (see Theorem 10) that the higher order Riesz transforms $\left(\delta^{\alpha}\right)^{k}\left(L_{\alpha}\right)^{-k / 2}$ are bounded in $L^{p}\left(y^{\delta} d y\right)$ for $\delta$ satisfying $\left(\mathrm{C}_{\alpha}\right)$, the "Sobolev" spaces $\mathcal{W}_{\alpha}^{k, p}\left(y^{\delta}\right)$ are different from the potential spaces $\mathfrak{W}_{\alpha, k}^{p}\left(y^{\delta}\right)$. In fact, we have the following

TheOREM 2. Let $1<p<\infty$.

(i) Let $\alpha>-1$ and let $\delta$ satisfy $\left(\mathrm{C}_{\alpha}\right)$. Then $\mathfrak{W}_{\alpha, k}^{p}\left(y^{\delta}\right) \subset \mathcal{W}_{\alpha}^{k, p}\left(y^{\delta}\right)$.

(ii) Let $-1<\alpha \leq 0$. Then $\mathfrak{W}_{\alpha, 2}^{2} \neq \mathcal{W}_{\alpha}^{2,2}$.

(iii) Let $\alpha>0$ and let $\delta$ satisfy $\left(\mathrm{C}_{\alpha-1}\right)$. Then $\mathfrak{W}_{\alpha, 2}^{p}\left(y^{\delta}\right)=\mathcal{W}_{\alpha}^{2, p}\left(y^{\delta}\right)$.

This result suggests that the iterations of the operators $\delta^{\alpha}$ are not good substitutes for the notion of fractional derivative in this case. Looking at the actual action of these operators on the set of eigenfunctions of the operator $L_{\alpha}$ (see (12) and (13)), it seems natural to consider the higher order Riesz transforms defined as

$$
R_{\alpha}^{k}=\left(\delta^{\alpha+k-1} \circ \cdots \circ \delta^{\alpha+1} \circ \delta^{\alpha}\right)\left(L_{\alpha}\right)^{-k / 2} .
$$

It is proved in Theorem 8 that they are bounded on $L^{p}\left((0, \infty), y^{\delta} d y\right)$ for $\delta$ satisfying $\left(\mathrm{C}_{\alpha}\right)$. This would suggest the following alternative concept of the "Sobolev" space given in Definition 3.

Definition 4. The Laguerre-Sobolev space, which we denote by $W_{\alpha}^{k, p}\left(y^{\delta}\right)$, is the set of functions $f$ in $L^{p}\left((0, \infty), y^{\delta} d y\right)$ such that

$$
\delta^{\alpha+m} \circ \cdots \circ \delta^{\alpha+1} \circ \delta^{\alpha} f \in L^{p}\left((0, \infty), y^{\delta} d y\right), \quad 0 \leq m \leq k-1,
$$

with the norm

$$
\|f\|_{W_{\alpha}^{k, p}\left(y^{\delta}\right)}=\|f\|_{p, \delta}+\sum_{m=0}^{k-1}\left\|\delta^{\alpha+m} \circ \cdots \circ \delta^{\alpha+1} \circ \delta^{\alpha} f\right\|_{p, \delta} .
$$

These spaces are the right spaces for the problem we are considering and the following theorem will be proved in Section 3.

Theorem 3. Let $k \in \mathbb{N}, \alpha>-1$, and let $1<p<\infty$ and $\delta$ satisfy $\left(\mathrm{C}_{\alpha}\right)$. Then

$$
W_{\alpha}^{k, p}\left(y^{\delta}\right)=\mathfrak{W}_{\alpha, k}^{p}\left(y^{\delta}\right),
$$

and the norms are equivalent.

Unweighted Sobolev spaces in the case of Hermite operators were considered previously by Thangavelu [21] and the authors [3]. 
For the case of Laguerre functions, Laguerre potential spaces were introduced by Peetre and Sparr in 1975; they were also studied by Thangavelu in [21] and by Radha and Thangavelu in [13] and [14]. For some previous work containing the definition and power weighted $L^{p}$-boundedness of the first order Riesz transforms, see [10] and [9] for the system $\mathcal{L}_{k}^{\alpha}$, and [12] for the system $\varphi_{k}^{\alpha}$ (see Section 5). Recently, power weighted $L^{p}$-boundedness of the higher order Riesz transforms of the form $\left(\mathbf{D}^{\alpha}\right)^{k} \mathbf{L}^{-k / 2}$ for the system $\varphi_{k}^{\alpha}$ (see Section 5) has been proved in [2]. From that result one can deduce, by using the methods in Section 5, our Theorem 10 about operators of the form $\left(\delta^{\alpha}\right)^{k}\left(L_{\alpha}\right)^{-k / 2}$. However, we present a different proof since we think that it contains some explanation of the commuting properties of several operators. Finally, for the case of Laguerre polynomials some results can be found in [8].

2. Hermite Sobolev spaces with weights. Let $H_{n}, n=0,1, \ldots$, be the family of Hermite polynomials. The Hermite function of order $n$ is defined as

$$
h_{n}(t)=\frac{H_{n}(t) e^{-t^{2} / 2}}{\left(2^{n} n ! \pi^{1 / 2}\right)^{1 / 2}}, \quad t \in \mathbb{R} .
$$

Given a multi-index $\alpha=\left(\alpha_{j}\right)_{j=1}^{d} \in \mathbb{N}^{d}$, the Hermite function of order $\alpha$ is defined as

$$
h_{\alpha}(x)=\prod_{j=1}^{d} h_{\alpha_{j}}\left(x_{j}\right), \quad x=\left(x_{1}, \ldots, x_{d}\right) \in \mathbb{R}^{d} .
$$

These functions are eigenvectors of the Hermite operator (see (1)). In fact,

$$
H h_{\alpha}=(2|\alpha|+d) h_{\alpha},
$$

where $|\alpha|=\sum_{j=1}^{d} \alpha_{j}$ (see $[20]$ ).

We shall need the following lemmas. Their proofs can be found respectively in [20], [17] and [3].

Lemma 1. Let $M \in \mathbb{N}$ and $f \in C_{c}^{\infty}$. Then there exists a constant $C_{M, f}>0$ such that

$$
\left|\int_{\mathbb{R}^{d}} f h_{\alpha}\right| \leq C_{M, f}(|\alpha|+1)^{-M}, \quad \alpha \in \mathbb{N}^{d} .
$$

Lemma 2. Let $1 \leq p<\infty$ and $w \in A_{p}$. Then there exist constants $\varepsilon_{p}>0$ and $C_{w}$ such that

$$
\left\|h_{\alpha}\right\|_{L^{p}(w)} \leq C_{w}(|\alpha|+1)^{\varepsilon_{p}} .
$$


Lemma 3. Let $f$ be a linear combination of Hermite functions. Then the fractional integral $H^{-s}, s>0$ (see (2)), has an integral representation

$$
H^{-s} f(x)=\int_{\mathbb{R}^{d}} K_{s}(x, y) f(y) d y, \quad x \in \mathbb{R}^{d},
$$

where $K_{s}(x, y)$ is positive and symmetric. Moreover,

$$
K_{s}(x, y) \leq C \phi_{s}(|x-y|), \quad x, y \in \mathbb{R}^{d},
$$

where $\phi_{s}(r)$, for $r \geq 0$, is defined by

$$
\phi_{s}(r)= \begin{cases}\chi_{\{r<1\}}(r) / r^{d-2 s}+e^{-r^{2} / 4} \chi_{\{r \geq 1\}}(r) & \text { if } s<d / 2, \\ \log (e / r) \chi_{\{r<1\}}(r)+e^{-r^{2} / 4} \chi_{\{r \geq 1\}}(r) & \text { if } s=d / 2, \\ \chi_{\{r<1\}}(r)+e^{-r^{2} / 4} \chi_{\{r \geq 1\}}(r) & \text { if } s>d / 2 .\end{cases}
$$

Theorem 4. Let $1 \leq p<\infty$ and $s>0$. If $w \in A_{p}$, then the operator $H^{-s}$ is bounded on $L^{p}(w)$.

Proof. If $p>1$, we just observe that the function $x \mapsto \phi_{s}(|x|)$ is radial and decreasing for $|x| \rightarrow \infty$; therefore, $\left|H^{-s} f(x)\right| \leq M(|f|)(x)$ where $M$ is the Hardy-Littlewood maximal operator, and the result follows.

In the case $p=1$, we shall prove that $\int_{\mathbb{R}^{d}} K_{s}(x, y) w(x) d x \leq C w(y)$ whenever $y$ is a Lebesgue point of $w$. Then

$$
\int_{\mathbb{R}^{d}}\left|H^{-s} f(x)\right| w(x) d x \leq \int_{\mathbb{R}^{d}}|f(y)| \int_{\mathbb{R}^{d}} K_{s}(x, y) w(x) d x d y \leq \int_{\mathbb{R}^{d}}|f(y)| w(y) d y .
$$

If $y$ is a Lebesgue point of $w \in A_{1}$, then

$$
\frac{1}{|B(y, r)|} \int_{B(y, r)} w(x) d x \leq C w(y) .
$$

Hence, by estimate (5) and splitting into annuli, we have

$$
\begin{aligned}
\int_{\mathbb{R}^{d}} K_{s}(x, y) w(x) d x & \leq C \sum_{k=-\infty}^{\infty} \int_{B\left(y, 2^{k}\right) \backslash B\left(y, 2^{k-1}\right)} \phi_{s}(|y-x|) w(x) d x \\
& \leq C \sum_{k=-\infty}^{\infty} \phi_{s}\left(2^{k}\right) \frac{2^{d k}}{\left|B\left(y, 2^{k}\right)\right|} \int_{B\left(y, 2^{k}\right) \backslash B\left(y, 2^{k-1}\right)} w(x) d x \\
& \leq C w(y)\left(\sum_{k=-\infty}^{\infty} \phi_{s}\left(2^{k}\right) 2^{d k}\right) \leq C w(y) .
\end{aligned}
$$

Given $1 \leq p<\infty, s>0$ and $w \in A_{p}$, we define the potential spaces

$$
\mathfrak{L}_{s}^{p}(w)=H^{-s / 2}\left(L^{p}(w)\right),
$$

with the norm $\|f\|_{\mathcal{L}_{s}^{p}(w)}=\|g\|_{L^{p}(w)}$, where $g$ is such that $H^{-s / 2} g=f$. The space $\mathfrak{L}_{s}^{p}(w)$ is well defined, since $H^{-s / 2}$ is bounded and one-to-one in $L^{p}(w)$. 
In fact, suppose $g \in L^{p}(w)$ and $H^{-s / 2} g=0$. Observe that

$$
\iint_{\mathbb{R}^{d}} K_{\mathbb{R}^{d}} K_{s / 2}(x, y)|g(x)|\left|h_{\alpha}(y)\right| d y d x \leq\left\|H^{-s / 2}|g|\right\|_{L^{p}(w)}\left\|h_{\alpha}\right\|_{L^{p^{\prime}\left(w^{\left.-p^{\prime} / p\right)}\right.}},
$$

and this expression is finite by Theorem 4 and Lemma 2 , since $w^{-p^{\prime} / p}$ belongs to $A_{p^{\prime}}$. Hence, by Fubini and the symmetry of $K_{s / 2}$,

$$
\int_{\mathbb{R}^{d}} g h_{\alpha}=(2 n+1)^{s / 2} \int_{\mathbb{R}^{d}} g H^{-s / 2} h_{\alpha}=(2 n+1)^{s / 2} \int_{\mathbb{R}^{d}} H^{-s / 2} g h_{\alpha}=0,
$$

and this yields $g=0$ (see Corollary 2.4 in [17]).

REMARK 1. The space $\mathfrak{F}$ of finite linear combinations of Hermite functions is a dense subspace of $\mathfrak{L}_{s}^{p}(w)$, since $\mathfrak{F}=H^{-s / 2}(\mathfrak{F})$ is dense in $L^{p}(w)$.

The operator $H$ can be factorized as

$$
H=\frac{1}{2} \sum_{j=1}^{d}\left(A_{j} A_{-j}+A_{-j} A_{j}\right),
$$

where

$$
A_{j}=\frac{\partial}{\partial x_{j}}+x_{j} \quad \text { and } \quad A_{-j}=-\frac{\partial}{\partial x_{j}}+x_{j} .
$$

It is easy to check that

$$
A_{j} h_{\alpha}=\sqrt{2 \alpha_{j}} h_{\alpha-e_{j}}, \quad A_{-j} h_{\alpha}=\sqrt{2\left(\alpha_{j}+1\right)} h_{\alpha+e_{j}},
$$

where $e_{j}$ is the $j$ th coordinate vector in $\mathbb{N}^{d}$. From these formulas the operators $A_{j}$ and $A_{j}^{*}$ are called annihilation and creation operators respectively.

Definition 5. The Hermite-Riesz transforms of order $m, m \in \mathbb{N}$, associated to $H$ are defined by

$R_{J}^{m}=A_{j_{1}} \ldots A_{j_{m}} H^{-m / 2}$, where $J=\left(j_{1}, \ldots, j_{m}\right), 1 \leq\left|j_{i}\right| \leq d, 1 \leq i \leq m$.

In the case $j_{1}=\cdots=j_{m}=j$, these operators will be denoted by $R_{j}^{m}$. The case $m=1$ was considered by S. Thangavelu (see [20]). He proved that these operators are bounded in $L^{p}\left(\mathbb{R}^{d}\right)$. Also in [17] and [18], it was shown that the operators $R_{J}^{m}$ are Calderón-Zygmund operators and as a consequence they are bounded in $L^{p}(w)$ for $w \in A_{p}, 1<p<\infty$.

We now present a structural theorem for the spaces $\mathfrak{L}_{s}^{p}(w)$. Unweighted versions of this result can be found in [3, Theorems 2, 6 and 7].

Theorem 5. Let $w \in A_{p}, 1<p<\infty$, and $s>0$.

(i) If $t>s$, then $\mathfrak{L}_{t}^{p}(w) \subset \mathfrak{L}_{s}^{p}(w) \subset L^{p}(w)$ with continuous inclusions. Moreover, $\mathfrak{L}_{s}^{p}(w)$ and $\mathfrak{L}_{t}^{p}(w)$ are isometrically isomorphic.

(ii) If $t>0$, then $H^{-t / 2}$ maps $\mathfrak{L}_{s}^{p}(w)$ into $\mathfrak{L}_{s+t}^{p}(w)$. 
(iii) If $s>1$ and $1 \leq|j| \leq d$, then $A_{j}$ is bounded from $\mathfrak{L}_{s}^{p}(w)$ into $\mathfrak{L}_{s-1}^{p}(w)$.

(iv) The operators $R_{J}^{m}$ are bounded on $\mathfrak{L}_{s}^{p}(w)$.

Proof. Observe that $H^{-t / 2}=H^{-s / 2} \circ H^{-r}$ with $r=(t-s) / 2$. Then (i) follows from Theorem 4; (ii) also follows from Theorem 4 and the definition of the spaces $\mathfrak{L}_{s}^{p}(w)$.

In order to prove (iii) we shall need the following two results. They can be found respectively in [7] and [3]. For further reference, we state them as Proposition 1 and Lemma 4.

Proposition 1. Let $1<p<\infty$ and $m \in \ell^{\infty}\left(\mathbb{N}^{d}\right)$ be such that

$$
\left|\Delta^{\ell} m(\alpha)\right| \leq C(1+|\alpha|)^{-|\ell|}, \quad \alpha \in \mathbb{N}^{d},|\ell| \leq d+1 .
$$

Consider the operator $\mathcal{T}_{m} f=\sum_{\alpha} m(\alpha)\left\langle f, h_{\alpha}\right\rangle h_{\alpha}$, defined at least for $f \in$ $L^{2}(\mathbb{R})$. Then $\mathcal{T}_{m}$ admits a bounded extension to $L^{p}(w)$ whenever the weight $w$ belongs to the Muckenhoupt class $A_{p}$.

REMARK 2. Observe that as $H h_{\alpha}=(2|\alpha|+d) h_{\alpha}$, any operator of the type $F(H) f=\sum_{\alpha} F(2 \alpha+d)\left\langle f, h_{\alpha}\right\rangle h_{\alpha}$ can be written as $\mathcal{T}_{m} f=$ $\sum_{\alpha} m(\alpha)\left\langle f, h_{\alpha}\right\rangle h_{\alpha}$ with $m(\alpha)=F(2 \alpha+d)=F\left(2\left(\alpha_{1}, \ldots, \alpha_{d}\right)+d\right)$.

Lemma 4. Let $b \in \mathbb{R}$. Then for all $f$ in $\mathfrak{F}$, we have

$$
\begin{array}{ll}
A_{j} H^{b} f=(H+2)^{b} A_{j} f, & 1 \leq j \leq d, \\
A_{j} H^{b} f=(H-2)^{b} A_{j} f, & -d \leq j \leq-1, \\
H^{b} A_{j} f=A_{j}(H-2)^{b} f, & 1 \leq j \leq d, \\
H^{b} A_{j} f=A_{j}(H+2)^{b} f, & -d \leq j \leq-1,
\end{array}
$$

where $H^{b} h_{\alpha}=(2|\alpha|+d)^{b} h_{n}$ and $(H+2)^{b} h_{\alpha}=(2|\alpha|+d+2)^{b} h_{\alpha}$ for all $\alpha \in \mathbb{N}_{0}^{d}$, and $(H-2)^{b} h_{\alpha}=(2|\alpha|+d-2)^{b} h_{\alpha}$ for all $\alpha$ with $|\alpha| \geq 1$.

We continue the proof of Theorem 5 . Let $1 \leq j \leq d$ (the case $-d \leq j \leq$ -1 is similar). Let $f \in \mathfrak{F}$. By Lemma 4 we have

$$
A_{j} f=H^{-(s-1) / 2}\left(\frac{H}{H+2}\right)^{(s-1) / 2} R_{j} H^{s / 2} f .
$$

As the function

$$
\mathbf{m}(\alpha)=\left(\frac{2|\alpha|+d}{2|\alpha|+d+2}\right)^{(s-1) / 2}
$$

satisfies the hypotheses of Proposition 1 (see also Remark 2), the operator $\left(\frac{H}{H+2}\right)^{(s-1) / 2}$ is bounded on $L^{p}(w)$. Hence by using the boundedness in $L^{p}(w)$ 
of the Riesz transforms, we have

$$
\begin{aligned}
\left\|A_{j} f\right\|_{\mathfrak{L}_{s-1}^{p}(w)} & =\left\|\left(\frac{H}{H+2}\right)^{(s-1) / 2} R_{j} H^{s / 2} f\right\|_{L^{p}(w)} \\
& \leq C\left\|H^{s / 2} f\right\|_{L^{p}(w)}=\|f\|_{\mathcal{L}_{s}^{p}(w)} .
\end{aligned}
$$

Finally, (iv) follows from (ii) and (iii).

The following technical result will be needed later.

Proposition 2. Let $1<p<\infty$ and $w \in A_{p}$. For $k \in \mathbb{N}$ the set $W^{k, p}(w)$ (see Definition 1) is a Banach space. Moreover, the sets $\mathfrak{F}$ and $C_{c}^{\infty}$ are dense in $W^{k, p}(w)$.

Proof. Observe that if $\left\{f_{n}\right\}_{n \geq 1}$ is a Cauchy sequence in $W^{k, p}(w)$, the completeness of $L^{p}(w)$ implies that $f_{n}$ converges to some $f$ and $A_{j}^{m} f_{n}$ converges to some $g_{m, j}$ in $L^{p}(w), 1 \leq m \leq k, 1 \leq j \leq d$. If $\psi$ belongs to $C_{c}^{\infty}$, also $\left(A_{j}^{m}\right)^{*} \psi$ belongs to $C_{c}^{\infty}$, and if $B$ is a ball containing the support of $\left(A_{j}^{m}\right)^{*} \psi$, then

$$
\begin{aligned}
\left|\int_{\mathbb{R}^{d}} f\left(A_{j}^{m}\right)^{*} \psi-\int_{\mathbb{R}^{d}} f_{n}\left(A_{j}^{m}\right)^{*} \psi\right| & \leq C \int_{B}\left|f-f_{n}\right| \\
& \leq C\left(\int_{\mathbb{R}^{d}}\left|f-f_{n}\right|^{p} w\right)^{1 / p}\left(\int_{B} w^{-p^{\prime} / p}\right)^{1 / p^{\prime}},
\end{aligned}
$$

where the last integral is finite since $w \in A_{p}$. Hence

$$
\lim _{n \rightarrow \infty} \int_{\mathbb{R}^{d}} f_{n}\left(A_{j}^{m}\right)^{*} \psi=\int_{\mathbb{R}^{d}} f\left(A_{j}^{m}\right)^{*} \psi .
$$

In the same way, $\lim _{n \rightarrow \infty} \int_{\mathbb{R}^{d}} A_{j}^{m} f_{n} \psi=\int_{\mathbb{R}^{d}} g_{m, j} \psi$. Therefore $\int_{\mathbb{R}^{d}} A_{j}^{m} f \psi=$ $\int_{\mathbb{R}^{d}} g_{m, j} \psi$ for all $\psi$ in $C_{c}^{\infty}$, and thus $A_{j}^{m} f=g_{m, j}$ almost everywhere. This completes the proof that $W^{k, p}(w)$ is complete.

Now we will see that $C_{c}^{\infty}$ is a dense set in $W^{k, p}(w)$ (we follow the ideas in $\left[15\right.$, p. 123]). Let $\psi \in C_{c}^{\infty}$ be such that $\int_{\mathbb{R}^{d}} \psi=1$. For every $\varepsilon>0$, consider $\psi_{\varepsilon}(x)=\left(1 / \varepsilon^{d}\right) \psi(x / \varepsilon)$. Given $f$ in $W^{1, p}(w)$, the function $f * \psi_{\varepsilon}$ belongs to $C^{\infty}$ and approximates $f$ in the $W^{1, p}(w)$-norm. In fact, it is easy to see that for all $m \geq 1$ and $1 \leq j \leq d$,

$$
A_{j}\left(f * \psi_{\varepsilon}\right)=A_{j} f * \psi_{\varepsilon}+\varepsilon f *\left(x_{j} \psi\right)_{\varepsilon}, \quad A_{j}^{m}\left(f * \psi_{\varepsilon}\right)=\sum_{n=0}^{m} \varepsilon^{n} A_{j}^{m-n} f *\left(x_{j}^{n} \psi\right)_{\varepsilon} .
$$

Since $A_{j}^{m-n} f$ belongs to $L^{p}(w)$ and $x_{j}^{n} \psi$ belongs to $C_{c}^{\infty}, 0 \leq n \leq m$, we have

$$
A_{j}^{m}\left(f * \psi_{\varepsilon}\right) \rightarrow A_{j}^{m} f
$$


in $L^{p}(w)$ as $\varepsilon$ goes to 0 . The functions $f * \psi_{\varepsilon}$ do not necessarily have compact support, but they can be modified as in the classical case (see [15, p. 123]).

It remains to prove that any function in $C_{c}^{\infty}$ can be approximated in the $W^{k, p}(w)$-norm by a function in $\mathfrak{F}$. We will show that any $f \in C_{c}^{\infty}$ is the limit, in the $W^{k, p}(w)$-norm, of a subsequence of partial sums

$$
S_{N} f=\sum_{|\alpha| \leq N}\left\langle f, h_{\alpha}\right\rangle h_{\alpha}, \quad N \geq 1,
$$

where $\left\langle f, h_{\alpha}\right\rangle=\int f h_{\alpha}$. In [17, Lemma 2.3], it is proved that there exists a subsequence of the previous sequence converging to $f$ in the $L^{p}(w)$-norm. Hence, it is enough to show that there exists a subsequence of

$$
\left\{A_{j}^{m}\left(S_{N}(f)\right)\right\}_{N \geq 1}=\left\{S_{N}\left(A_{j}^{m} f\right)\right\}_{N \geq 1}
$$

converging to $A_{j}^{m} f$ in the $L^{p}(w)$-norm, where $1 \leq j \leq d$ and $1 \leq m \leq k$.

Fix $j$ and $m$ such that $1 \leq j \leq d$ and $1 \leq m \leq k$. Following the argument of [17], the sequence $\left\{S_{N}\left(A_{j}^{m} f\right)\right\}_{N \geq 1}$ converges to $A_{j}^{m} f$ in the $L^{2}$-norm. Hence we can take a subsequence $\left\{S_{N_{k}}\left(A_{j}^{m} f\right)\right\}_{k \geq 1}$ converging to $A_{j}^{m} f$ almost everywhere. By using (8), we have

$$
\begin{aligned}
S_{N}\left(A_{j}^{m} f\right) & =\sum_{|\alpha| \leq N}\left\langle A_{j}^{m} f, h_{\alpha}\right\rangle h_{\alpha}=\sum_{|\alpha| \leq N}\left\langle f,\left(A_{j}^{*}\right)^{m} h_{\alpha}\right\rangle h_{\alpha} \\
& =\sum_{|\alpha| \leq N} \prod_{l=1}^{m} \sqrt{2\left(\alpha_{i}+l\right)}\left\langle f, h_{\alpha+m e_{i}}\right\rangle h_{\alpha} .
\end{aligned}
$$

Hence, by Lemma 1 (with $M \geq m$ ) and Hölder's inequality, we have

$$
\begin{aligned}
\left|S_{N}\left(A_{j}^{m} f\right)\right|^{p} & \leq C\left(\sum_{|\alpha| \leq N} \prod_{l=1}^{m} \sqrt{2\left(\alpha_{j}+l\right)}(|\alpha|+m+1)^{-M}\left|h_{\alpha}\right|\right)^{p} \\
& \leq C\left(\sum_{|\alpha| \leq N}(|\alpha|+m+1)^{-M / 2}\left|h_{\alpha}\right|\right)^{p} \\
& \leq C\left(\sum_{\alpha}(|\alpha|+1)^{-M / 2}\right)^{p / p^{\prime}} \sum_{\alpha}(|\alpha|+1)^{-M / 2}\left|h_{\alpha}\right|^{p} \\
& \leq C \sum_{\alpha}(|\alpha|+1)^{-M / 2}\left|h_{\alpha}\right|^{p} .
\end{aligned}
$$

From Lemma 2, for $M$ large enough, the function

$$
\sum_{\alpha}(\alpha+1)^{-M / 2}\left|h_{\alpha}\right|^{p}
$$

belongs to $L^{1}(w)$. The dominated convergence theorem implies that

$$
\left\{S_{N_{k}}\left(A_{j}^{m} f\right)\right\}_{k \geq 1} \rightarrow A_{j}^{m} f
$$


in the $L^{p}(w)$-norm. Now we can repeat the lines above for every $j$ and $m$, taking a subsequence of the previous subsequence in each step.

Proof of Theorem 1. Since $\mathfrak{F}$ is dense in both spaces, it is enough to show the equivalence of the norms for functions in $\mathfrak{F}$.

Let $f \in \mathfrak{F}$, and $f=H^{-k / 2} g$. For $1 \leq j \leq d$ and $1 \leq m \leq k$, from the boundedness of $R_{j}^{m}$ and $H^{-k+m}$ (see the comments after Definition 5 and Theorem 4) we have

$$
\left\|\left(A_{j}\right)^{m} f\right\|_{L^{p}(w)} \leq\left\|R_{j}^{m} H^{(-k+m / 2)} g\right\|_{L^{p}(w)} \leq\|g\|_{L^{p}(w)},
$$

so

$$
\|f\|_{W^{k, p}(w)} \leq C\|g\|_{L^{p}(w)}=C\|f\|_{\mathfrak{L}_{k}^{p}(w)} .
$$

Now we shall prove the reverse inequality. By using Lemma 4, the following identities can be proved for each integer $k \geq 1$ :

$$
\begin{aligned}
T_{k} & =\sum_{j=1}^{d} R_{-j}^{k} R_{j}^{k}=\sum_{j=1}^{d}\left(A_{j}^{*}\right)^{k} H^{-k / 2}\left(A_{j}\right)^{k} H^{-k / 2} \\
& =(H-2 k)^{-k / 2}\left(\sum_{j=1}^{d}\left(A_{j}^{*}\right)^{k}\left(A_{j}\right)^{k}\right) H^{-k / 2} \\
& =(H-2 k)^{-k / 2}\left\{\sum_{j=1}^{d}\left(\left(H_{j}-1\right)\left(H_{j}-1-2\right) \cdots\left(H_{j}-1-2(k-1)\right)\right)\right\} H^{-k / 2},
\end{aligned}
$$

where $H_{j}=-\partial^{2} / \partial x_{j}^{2}+x_{j}^{2}$. Observe that $H=\sum_{j} H_{j}$. Consider the function

$$
\mathbf{m}_{k}(\alpha)=\frac{(2|\alpha|+d-2 k)^{k / 2}(2|\alpha|+d)^{k / 2}}{\sum_{j=1}^{d}\left(2 \alpha_{j}\right)\left(2 \alpha_{j}-2\right) \ldots\left(2 \alpha_{j}-2(k-1)\right)} \chi_{[d k, \infty)}(|\alpha|) .
$$

An appropriate smooth extension of $\mathbf{m}_{k}$ can be considered in order to apply Proposition 1. Hence the operator $\mathcal{S}_{\mathbf{m}_{k}}$ defined as $\mathcal{S}_{\mathbf{m}_{k}} f=\sum_{\alpha} \mathbf{m}_{k}(\alpha)\left\langle f, h_{\alpha}\right\rangle h_{\alpha}$ is bounded in $L^{p}(w)$.

Denote by $\mathfrak{F}_{k}$ the finite-dimensional space of linear combinations of Hermite functions $h_{\alpha}$ with $|\alpha|<k$. Given a function $g$ in $\mathfrak{F} \backslash \mathfrak{F}_{k}$, we observe that $\mathcal{S}_{\mathbf{m}_{k}} \circ T_{k} g=g$ and therefore

$$
\begin{aligned}
\|g\|_{L^{p}(w)} & =\left\|\mathcal{S}_{\mathbf{m}_{k}} T_{k} g\right\|_{L^{p}(w)} \leq C_{k}\left\|T_{k} g\right\|_{L^{p}(w)}=C_{k} \sum_{j=1}^{d}\left\|R_{-j}^{k} R_{j}^{k} g\right\|_{L^{p}(w)} \\
& \leq C_{k} \sum_{j=1}^{d}\left\|R_{j}^{k} g\right\|_{L^{p}(w)}=C_{k} \sum_{j=1}^{d}\left\|\left(A_{j}\right)^{k} H^{-k / 2} g\right\|_{L^{p}(w)}
\end{aligned}
$$

for some constant $C_{k}$ independent of $g$. Therefore for $f \in \mathfrak{F} \backslash \mathfrak{F}_{k}$ with $f=$ 
$H^{-k / 2} g$, we have

$$
\|f\|_{\mathcal{L}_{k}^{p}(w)}=\|g\|_{L^{p}(w)} \leq C_{k}\|f\|_{W^{k, p}(w)} .
$$

For the general case $g \in \mathfrak{F}$, we write $g=g_{1}+g_{2}$ with $g_{1} \in \mathfrak{F}_{k}$ and $g_{2} \in \mathfrak{F} \backslash \mathfrak{F}_{k}$. We observe (one can use Lemma 2) that $H^{k / 2}$ is a bounded linear operator on the finite-dimensional space $\mathfrak{F}_{k}$ (with the $L^{p}(w)$-norm). The same lemma also ensures that the projection $g \mapsto g_{1}$ is bounded in $L^{p}(w)$, hence

$$
\begin{aligned}
\|g\|_{L^{p}(w)} & \leq\left\|H^{k / 2} H^{-k / 2} g_{1}\right\|_{L^{p}(w)}+\left\|g_{2}\right\|_{L^{p}(w)} \\
& \leq C_{k}\left\|H^{-k / 2} g_{1}\right\|_{L^{p}(w)}+C_{k} \sum_{j=1}^{d}\left\|\left(A_{j}\right)^{k} H^{-k / 2} g_{2}\right\|_{L^{p}(w)} \\
& \leq C\left\|H^{-k / 2} g\right\|_{L^{p}(w)}+C_{k} \sum_{j=1}^{d}\left\|\left(A_{j}\right)^{k} H^{-k / 2} g\right\|_{L^{p}(w)},
\end{aligned}
$$

where in the last inequality we have used $\left(A_{j}\right)^{k} H^{k / 2} g_{1}=0$ and the fact that the projection of the function $H^{-k / 2} g$ from $\mathfrak{F}$ into $\mathfrak{F}_{k}$ is $H^{-k / 2} g_{1}$.

3. Laguerre setting. Let $L_{n}^{\alpha}, n=0,1, \ldots$, be the Laguerre polynomials of type $\alpha>-1$. Consider the family of Laguerre functions $\mathcal{L}_{n}^{\alpha}$ defined as

$$
\mathcal{L}_{n}^{\alpha}(y)=\left(\frac{\Gamma(n+1)}{\Gamma(n+\alpha+1)}\right)^{1 / 2} e^{-y / 2} y^{\alpha / 2} L_{n}^{\alpha}(y), \quad y \in \mathbb{R}^{+}, n \in \mathbb{N}_{0} .
$$

For each $\alpha>-1,\left\{\mathcal{L}_{n}^{\alpha}\right\}_{n=0}^{\infty}$ is an orthonormal system in $L^{2}((0, \infty))$ and satisfies

$$
L_{\alpha} \mathcal{L}_{n}^{\alpha}=\left(n+\frac{\alpha+1}{2}\right) \mathcal{L}_{n}^{\alpha}, \quad n \in \mathbb{N}_{0},
$$

where $L_{\alpha}$ is defined in (3). It is known (and probably belongs to the folklore, see for example [19, Theorem 5.7.1]) that if $\alpha>-1$, and $1<p<\infty$ and $\delta \in \mathbb{R}$ satisfy $\left(\mathrm{C}_{\alpha}\right)$ (see (4)), then the set $S_{\alpha}$ of finite linear combinations of Laguerre functions is dense in $L^{p}\left((0, \infty), y^{\delta} d y\right)$. The condition $\left(\mathrm{C}_{\alpha}\right)$ will be crucial along this note.

REMARK 3. Observe that if a pair $(\delta, p)$ satisfies $\left(\mathrm{C}_{\alpha}\right)$ then it satisfies $\left(\mathrm{C}_{\beta}\right)$ for every $\beta>\alpha$.

Given $\alpha>-1$ and $s>0$, we can define the operator $\left(L_{\alpha}\right)^{-s}$ analogously to (2) just by replacing $\left\{e^{-t H}\right\}_{t>0}$ by $\left\{e^{-t L_{\alpha}}\right\}_{t>0}$. We need the following two results that can be found in [7] and that we state as a unified theorem for further reference.

TheOREM 6. Let $\alpha>-1$, and let $1<p<\infty$ and $\delta \in \mathbb{R}$ satisfy condition $\left(\mathrm{C}_{\alpha}\right)$. Let $\mu \in C^{\infty}([0, \infty))$ be such that

$$
\left|\mu^{(k)}(t)\right| \leq C_{k}(1+t)^{-k}, \quad k=0,1,2, \ldots,
$$


for all $t>0$ and $k \in \mathbb{N}_{0}$. Then the operator

$$
T_{\mu} f=\sum_{n=0}^{\infty} \mu(n)\left\langle f, \mathcal{L}_{n}^{\alpha}\right\rangle \mathcal{L}_{n}^{\alpha},
$$

defined at least for $f \in L^{2}(\mathbb{R})$, admits a bounded extension to $L^{p}\left((0, \infty), y^{\delta} d y\right)$.

A consequence of this result is the following theorem.

TheOREM 7. Let $\alpha>-1$, and let $1<p<\infty$ and $\delta \in \mathbb{R}$ satisfy condition $\left(\mathrm{C}_{\alpha}\right)$. Then the operator $\left(L_{\alpha}\right)^{-s}, s>0$, is bounded from $L^{p}\left((0, \infty), y^{\delta} d y\right)$ into itself.

Proof. The multiplier $\mu(n)=(n+(\alpha+1) / 2)^{-s}$ satisfies (10).

Now that we see that the spaces in Definition 2 are well defined, we proceed as in the Hermite context. It is not difficult to prove that $\left(L_{\alpha}\right)^{-s / 2}$ is one-to-one in $L^{p}\left((0, \infty), y^{\delta} d y\right)$, using the fact that $S_{\alpha}$ is contained and dense in $L^{p^{\prime}}\left((0, \infty), y^{-p^{\prime} \delta / p} d y\right)$ whenever $\delta$ satisfies $\left(\mathrm{C}_{\alpha}\right)$. Moreover, since $S_{\alpha}=$ $\left(L_{\alpha}\right)^{-s / 2}\left(S_{\alpha}\right)$ and $S_{\alpha}$ is dense in $L^{p}\left((0, \infty), y^{\delta} d y\right)$, it is dense in $\mathfrak{W}_{\alpha, s}^{p}\left(y^{\delta}\right)$.

The operator $L_{\alpha}$ can be written as

$$
L_{\alpha}=\left(\delta^{\alpha}\right)^{*} \delta^{\alpha}+(\alpha+1) / 2,
$$

where

$$
\delta^{\alpha}=\sqrt{x} \frac{d}{d x}+\frac{1}{2}\left(\sqrt{x}-\frac{\alpha}{\sqrt{x}}\right), \quad\left(\delta^{\alpha}\right)^{*}=-\sqrt{x} \frac{d}{d x}+\frac{1}{2}\left(\sqrt{x}-\frac{\alpha+1}{\sqrt{x}}\right) .
$$

The action of these operators on Laguerre functions is given by

$$
\begin{aligned}
& \delta^{\alpha}\left(\mathcal{L}_{0}^{\alpha}\right)=0, \quad \delta^{\alpha}\left(\mathcal{L}_{n}^{\alpha}\right)=-\sqrt{n} \mathcal{L}_{n-1}^{\alpha+1}, \quad \text { for } n \geq 1, \\
& \left(\delta^{\alpha}\right)^{*}\left(\mathcal{L}_{n}^{\alpha+1}\right)=-\sqrt{n+1} \mathcal{L}_{n+1}^{\alpha} \quad \text { for } n \geq 0 .
\end{aligned}
$$

The Riesz transforms were defined in [10] for $\alpha>-1$ by

$$
R_{\alpha}=\delta^{\alpha}\left(L_{\alpha}\right)^{-1 / 2} \quad \text { and } \quad \widetilde{R}_{\alpha}=\left(\delta^{\alpha}\right)^{*}\left(L_{\alpha+1}\right)^{-1 / 2} .
$$

In [9] it was proved that those operators are bounded on $L^{p}\left((0, \infty), y^{\delta} d y\right)$ for $\delta$ satisfying $\left(\mathrm{C}_{\alpha}\right)$. Given a positive integer $k$ and $\alpha>-1$ we define the higher order Riesz transforms of order $k$ as

$$
R_{\alpha}^{k}=\left(\delta^{\alpha+k-1} \circ \cdots \circ \delta^{\alpha+1} \circ \delta^{\alpha}\right)\left(L_{\alpha}\right)^{-k / 2}
$$

and

$$
\widetilde{R}_{\alpha}^{k}=\left(\left(\delta^{\alpha}\right)^{*} \circ\left(\delta^{\alpha+1}\right)^{*} \circ \cdots \circ\left(\delta^{\alpha+k-1}\right)^{*}\right)\left(L_{\alpha+k}\right)^{-k / 2} .
$$

Observe that $R_{\alpha}^{1}=R_{\alpha}$ and $\widetilde{R}_{\alpha}^{1}=\widetilde{R}_{\alpha}$.

Theorem 8. Let $k \in \mathbb{N}, \alpha>-1$ and let $1<p<\infty$ and $\delta$ satisfy $\left(\mathrm{C}_{\alpha}\right)$. Then the operators $R_{\alpha}^{k}$ and $\widetilde{R}_{\alpha}^{k}$ are bounded on $L^{p}\left((0, \infty), y^{\delta} d y\right)$.

In order to prove this theorem we shall need the following lemma, whose proof is left to the reader. 
Lemma 5. Let $\Phi$ be a continuous function and $\alpha>-1$. For every $f$ in $S_{\alpha}$, we have

(1) $\delta^{\alpha} \Phi\left(L_{\alpha}\right) f=\Phi\left(L_{\alpha+1}+\frac{1}{2} I\right) \delta^{\alpha} f$.

(2) $\left(\delta^{\alpha}\right)^{*} \Phi\left(L_{\alpha+1}\right) f=\Phi\left(L_{\alpha}-\frac{1}{2} I\right)\left(\delta^{\alpha}\right)^{*} f$.

Now we can prove Theorem 8 by induction on $k$.

Proof of Theorem 8. As mentioned above, the result is true for $k=1$ (see [9]). Let $k>1$. For a function $f$ in $S_{\alpha}$, we have

$$
\begin{aligned}
R_{\alpha}^{k} & =\left(\delta^{\alpha+k-1} \circ \cdots \circ \delta^{\alpha+1} \circ \delta^{\alpha}\right)\left(L_{\alpha}\right)^{-k / 2} \\
& =\left(\delta^{\alpha+k-1} \circ \cdots \circ \delta^{\alpha+1}\right) \circ \delta^{\alpha} \circ\left(L_{\alpha}\right)^{-(k-1) / 2}\left(L_{\alpha}\right)^{-1 / 2} \\
& =\left(\delta^{\alpha+k-1} \circ \cdots \circ \delta^{\alpha+1}\right) \circ\left(L_{\alpha+1}+\frac{1}{2} I\right)^{-(k-1) / 2} \circ \delta^{\alpha} \circ\left(L_{\alpha}\right)^{-k / 2} \\
& =\left(\delta^{\alpha+k-1} \circ \cdots \circ \delta^{\alpha+1}\right) \circ\left(L_{\alpha+1}\right)^{-(k-1) / 2} \circ T_{\mu} \circ \delta^{\alpha} \circ\left(L_{\alpha}\right)^{-k / 2} \\
& =R_{\alpha+1}^{k-1} \circ T_{\mu} \circ R_{\alpha},
\end{aligned}
$$

where $T_{\mu}$ is the operator given by the multiplier (in the system $\left\{\mathcal{L}_{k}^{\alpha+1}\right\}_{k=0}^{\infty}$ )

$$
\mu(n)=\left[\frac{n+(\alpha+1) / 2}{n+(\alpha+2) / 2+1 / 2}\right]^{(k-1) / 2} .
$$

The function $\mu$ satisfies (10). Hence, by Theorem $6, T_{\mu}$ is bounded from $L^{p}\left((0, \infty), y^{\delta} d y\right)$ into $L^{p}\left((0, \infty), y^{\delta} d y\right)$ for $\delta$ satisfying $\left(\mathrm{C}_{\alpha+1}\right)$. On the other hand, the induction hypothesis says that $R_{\alpha+1}^{k-1}$ is bounded from $L^{p}\left((0, \infty), y^{\delta} d y\right)$ into $L^{p}\left((0, \infty), y^{\delta} d y\right)$ for $\delta$ satisfying $\left(\mathrm{C}_{\alpha+1}\right)$. As we noticed, this range is bigger than the range $\left(\mathrm{C}_{\alpha}\right)$ (see Remark 3 ).

To prove the boundedness of $\widetilde{R}_{\alpha}^{k}$, we again use Lemma 5 . We write $\widetilde{R}_{\alpha}^{k}=$ $\widetilde{R}_{\alpha}^{k-1} \circ T_{\nu} \circ \widetilde{R}_{\alpha+k-1}$, where $T_{\nu}$ is given by the multiplier

$$
\nu(n)=\left(n+\frac{\alpha+k}{2}\right) /\left(n+\frac{\alpha+k-1}{2}\right) .
$$

The proof continues along the same lines as for $R_{\alpha}^{k}$, by using the boundedness of the operators $\widetilde{R}_{\alpha}^{k}$ and $T_{\nu}$.

Parallel to the Hermite setting we have the following structural theorem for the spaces $\mathfrak{W}_{\alpha, s}^{p}\left(y^{\delta}\right)$.

Theorem 9. Let $s>0, \alpha>-1$, and let $1<p<\infty$ and $\delta$ satisfy $\left(\mathrm{C}_{\alpha}\right)$.

(i) Ift $>s$, then $\mathfrak{W}_{\alpha, s}^{p}\left(y^{\delta}\right) \subset \mathfrak{W}_{\alpha, t}^{p}\left(y^{\delta}\right) \subset L^{p}\left(y^{\delta}\right)$ with continuous inclusions. Moreover, $\mathfrak{W}_{\alpha, s}^{p}\left(y^{\delta}\right)$ and $\mathfrak{W}_{\alpha, t}^{p}\left(y^{\delta}\right)$ are isometrically isomorphic.

(ii) If $t>0$, then $\left(L_{\alpha}\right)^{-t / 2}$ maps $\mathfrak{W}_{\alpha, s}^{p}\left(y^{\delta}\right)$ into $\mathfrak{W}_{\alpha, s+t}^{p}\left(y^{\delta}\right)$.

(iii) If $s>1$, then $\delta^{\alpha}$ is bounded from $\mathfrak{W}_{\alpha, s}^{p}\left(y^{\delta}\right)$ into $\mathfrak{W}_{\alpha+1, s-1}^{p}\left(y^{\delta}\right)$.

(iv) The operators $R_{\alpha}^{k}$ are bounded from $\mathfrak{W}_{\alpha, s}^{p}\left(y^{\delta}\right)$ into $\mathfrak{W}_{\alpha+k, s}^{p}\left(y^{\delta}\right)$. 
Proof. The statements (i) and (ii) follow from the boundedness of $\left(L_{\alpha}\right)^{-s / 2}$ established in Theorem 7. On the other hand, given $f \in \mathfrak{W}_{\alpha, s}^{p}\left(y^{\delta}\right)$, there exists $g \in L^{p}\left(y^{\delta}\right)$ such that $L_{\alpha}^{-s / 2} g=f$. Set

Then

$$
h=\left(\frac{L_{\alpha+1}+\frac{1}{2} I}{L_{\alpha+1}}\right)^{-(s-1) / 2} R_{\alpha} g .
$$

$$
\begin{aligned}
\delta^{\alpha} f & =\delta^{\alpha} L_{\alpha}^{-s / 2} g=\left(L_{\alpha+1}+\frac{1}{2} I\right)^{-(s-1) / 2} \delta^{\alpha} L_{\alpha}^{-1 / 2} g \\
& =\left(L_{\alpha+1}+\frac{1}{2} I\right)^{-(s-1) / 2} R_{\alpha} g=\left(L_{\alpha+1}\right)^{-(s-1) / 2} h .
\end{aligned}
$$

By Theorems 6 and 8, we have

$$
\left\|\delta^{\alpha} f\right\|_{\mathfrak{W}_{\alpha+1, s-1}^{p}\left(y^{\delta}\right)}=\|h\|_{L^{p}\left(y^{\delta}\right)} \leq C\|g\|_{L^{p}\left(y^{\delta}\right)}=\left\|\delta^{\alpha} f\right\|_{\mathfrak{W}_{\alpha, s}^{p}\left(y^{\delta}\right)} .
$$

In order to prove (iv) we use (ii) and (iii).

Given a function $f$, consider the Cesàro sums of $g$ of order $r>0$, that is,

for $N \in \mathbb{N}$, with

$$
C_{N, \alpha}^{r}(g)=\frac{1}{\mathfrak{a}_{N}^{r}} \sum_{n=0}^{N} \mathfrak{a}_{N-n}^{r}\left\langle f, \mathcal{L}_{n}^{\alpha}\right\rangle \mathcal{L}_{n}^{\alpha}
$$

$$
\mathfrak{a}_{n}^{r}=\frac{\prod_{j=1}^{n}(j+r)}{n !}, \quad 0 \leq n \leq N .
$$

The following proposition is an easy consequence of Theorem 1.13 in [11] and it will be the key to proving a density result in $W_{\alpha}^{k, p}\left(y^{\delta}\right)$ (see Definition 4).

Proposition 3. Let $\alpha>-1$, and let $1<p<\infty$ and $\delta$ satisfy $\left(\mathrm{C}_{\alpha}\right)$. Then there exists $r \geq 1$ (possibly depending on $\alpha$ ) such that the Cesàro sums of order $r$ of a function $f$ converge to $f$ in the $L^{p}\left(y^{\delta} d y\right)$-norm as $N$ goes to infinity.

Proposition 4. Let $k \in \mathbb{N}, \alpha>-1$, and let $1<p<\infty$ and $\delta$ satisfy $\left(\mathrm{C}_{\alpha}\right)$. Then $S_{\alpha}$ is a dense subspace of $W_{\alpha}^{k, p}\left(y^{\delta}\right)$.

Proof. By using (12) and (13), we have

$$
\begin{aligned}
\delta^{\alpha} C_{N, \alpha}^{r}(f) & =\frac{1}{\mathfrak{a}_{N}^{r}} \sum_{n=1}^{N} \mathfrak{a}_{N-n}^{r}\left\langle f, \mathcal{L}_{n}^{\alpha}\right\rangle(-\sqrt{n}) \mathcal{L}_{n-1}^{\alpha+1} \\
& =\frac{1}{\mathfrak{a}_{N}^{r}} \sum_{n=1}^{N} \mathfrak{a}_{N-n}^{r}\left\langle f,\left(\delta^{\alpha}\right)^{*} \mathcal{L}_{n-1}^{\alpha+1}\right\rangle \mathcal{L}_{n-1}^{\alpha+1} \\
& =\frac{\mathfrak{a}_{N-1}^{r}}{\mathfrak{a}_{N}^{r}} \frac{1}{\mathfrak{a}_{N-1}^{r}} \sum_{n=0}^{N-1} \mathfrak{a}_{(N-1)-n}^{r}\left\langle\delta^{\alpha} f, \mathcal{L}_{n}^{\alpha+1}\right\rangle \mathcal{L}_{n}^{\alpha+1} \\
& =\frac{\mathfrak{a}_{N-1}^{r}}{\mathfrak{a}_{N}^{r}} C_{N-1, \alpha+1}^{r}\left(\delta^{\alpha} f\right),
\end{aligned}
$$


and inductively, if $m \in \mathbb{N}_{0}$ and $N>m$,

$$
\begin{aligned}
& \left(\delta^{\alpha+m-1} \circ \ldots \circ \delta^{\alpha+1} \circ \delta^{\alpha}\right) C_{N, \alpha}^{r}(f) \\
& \qquad \quad=\frac{\mathfrak{a}_{N-m}^{r}}{\mathfrak{a}_{N}^{r}} C_{N-m, \alpha+m}^{r}\left(\delta^{\alpha+m-1} \circ \cdots \circ \delta^{\alpha+1} \circ \delta^{\alpha} f\right) .
\end{aligned}
$$

By Proposition 3, we choose $r$ large enough so that the sequence in $S_{\alpha}$ given by $f_{N}=C_{N, \alpha}^{r} f$ converges to $f$ in the $L^{p}\left(y^{\delta} d y\right)$-norm. Observe that the functions $\delta^{\alpha+m-1} \circ \cdots \circ \delta^{\alpha+1} \circ \delta^{\alpha} f$, where $1 \leq m \leq k-1$, also belong to $L^{p}\left((0, \infty), y^{\delta} d y\right)$. Thus formula (14), Proposition 3 and the fact that $\lim _{N \rightarrow \infty} \mathfrak{a}_{N-m}^{r} / \mathfrak{a}_{N}^{r}=1$ imply that the sequence $\delta^{\alpha+m-1} \circ \cdots \circ \delta^{\alpha+1} \circ \delta^{\alpha} \circ$ $C_{N, \alpha}^{r}(f)$ converges to $\delta^{\alpha+m-1} \circ \cdots \circ \delta^{\alpha+1} \circ \delta^{\alpha} f$ in the $L^{p}\left(y^{\delta} d y\right)$-norm for $1 \leq m \leq k-1$.

Proof of Theorem 3. As $S_{\alpha}$ is a dense subspace of $\mathfrak{W}_{\alpha, k}^{p}\left(y^{\delta}\right)$ and $W_{\alpha}^{k, p}\left(y^{\delta}\right)$, it is enough to show the equivalence of the norms for functions $f \in S_{\alpha}$. Let $g$ be such that $\left(L_{\alpha}\right)^{-k / 2} g=f$. For $0 \leq m \leq k-1$, we have

$$
\begin{aligned}
\|f\|_{W_{\alpha}^{k, p}\left(y^{\delta}\right)} & =\|f\|_{p, \delta}+\sum_{m=1}^{k-1}\left\|\delta^{\alpha+m} \circ \cdots \circ \delta^{\alpha+1} \circ \delta^{\alpha} f\right\|_{p, \delta} \\
& =\left\|\left(L_{\alpha}\right)^{-k / 2} g\right\|_{p, \delta}+\sum_{m=1}^{k-1}\left\|\delta^{\alpha+m} \circ \cdots \circ \delta^{\alpha+1} \circ \delta^{\alpha}\left(L_{\alpha}\right)^{-k / 2} g\right\|_{p, \delta} \\
& =\left\|\left(L_{\alpha}\right)^{-k / 2} g\right\|_{p, \delta}+\sum_{m=1}^{k-1}\left\|R_{\alpha}^{m}\left(L_{\alpha}\right)^{-(k-m) / 2} g\right\|_{p, \delta} \\
& \leq C\|g\|_{p, \delta}=C\|f\|_{\mathfrak{W}_{\alpha, k}^{p}\left(y^{\delta}\right)} .
\end{aligned}
$$

In the last inequality we have used Theorems 7 and 8 .

For the reverse inequality it is clearly enough to prove that there exists a constant $C$ such that for all $f \in S_{\alpha}$,

$$
\left\|\left(L_{\alpha}\right)^{k / 2} f\right\|_{p, \delta} \leq C\left(\|f\|_{p, \delta}+\left\|\delta^{\alpha+k-1} \circ \cdots \circ \delta^{\alpha+1} \circ \delta^{\alpha} f\right\|_{p, \delta}\right) .
$$

Let $\alpha>-1$ and $k \in \mathbb{N}$. We let $\Pi_{\alpha}^{k}$ be the set of linear combinations of Laguerre functions of type $\alpha$ up to order $k$. If $f \in S_{\alpha}$, we split $f=f_{1}+f_{2}$ with $f_{1} \in \Pi_{\alpha}^{k}$ and $f_{2} \in S_{\alpha} \backslash \Pi_{\alpha}^{k}$. Since $\left(L_{\alpha}\right)^{k}$ is a linear operator on a finite-dimensional space $\Pi_{\alpha}^{k}$, there exists a constant $C$ that depends on $k$ such that

$$
\left\|\left(L_{\alpha}\right)^{k / 2} f_{1}\right\|_{p, \delta} \leq C\left\|f_{1}\right\|_{p, \delta} .
$$

On the other hand, since $\left(L_{\alpha}\right)^{-k / 2}$ is bounded on $L^{p}\left((0, \infty), y^{\delta} d y\right.$ ) (Theorem 7), we have 


$$
\begin{aligned}
\left\|f_{1}\right\|_{p, \delta} & =\left\|f-f_{2}\right\|_{p, \delta} \leq\|f\|_{p, \delta}+\left\|\left(L_{\alpha}\right)^{-k / 2}\left(L_{\alpha}\right)^{k / 2} f_{2}\right\|_{p, \delta} \\
& \leq\|f\|_{p, \delta}+C\left\|\left(L_{\alpha}\right)^{k / 2} f_{2}\right\|_{p, \delta},
\end{aligned}
$$

thus

$$
\left\|\left(L_{\alpha}\right)^{k / 2} f\right\|_{p, \delta} \leq\left\|\left(L_{\alpha}\right)^{k / 2} f_{1}\right\|_{p, \delta}+\left\|\left(L_{\alpha}\right)^{k / 2} f_{2}\right\|_{p, \delta} \leq C\left(\|f\|_{p, \delta}+\left\|\left(L_{\alpha}\right)^{k / 2} f_{2}\right\|_{p, \delta}\right) .
$$

Therefore, it is enough to prove (15) for $f_{2}$. By using Lemma 5 we can easily show the following identity for each integer $k$ :

$$
\begin{aligned}
T_{k}= & \widetilde{R}_{\alpha}^{k} \circ R_{\alpha}^{k} \\
= & \left(L_{\alpha}-\frac{k}{2}\right)^{-k / 2} \circ\left(L_{\alpha}-\frac{\alpha+1}{2}-k-1\right) \circ\left(L_{\alpha}-\frac{\alpha+1}{2}-k-2\right) \cdots \\
& \ldots \circ\left(L_{\alpha}-\frac{\alpha+1}{2}-1\right) \circ\left(L_{\alpha}-\frac{\alpha+1}{2}\right)\left(L_{\alpha}\right)^{-k / 2}
\end{aligned}
$$

and consider the function

$$
\mu_{k}(t)=\frac{\left(t+\frac{\alpha+1}{2}\right)^{k / 2}\left(t+\frac{\alpha+1}{2}-\frac{k}{2}\right)^{k / 2}}{\prod_{j=0}^{k-1}(t-j)} \chi_{[k, \infty)}(t),
$$

which satisfies (10). Then the proof follows the same lines as in the Hermite case in Theorem 3 using the multiplier Theorem 6 and Theorem 8 in order to control the operator $\widetilde{R}_{\alpha}^{k}$.

\section{Alternative definitions of Riesz transforms. Consequences}

for Sobolev spaces. In this section we analyse the role of the "natural" Riesz transforms

$$
\left(\delta^{\alpha}\right)^{k}\left(L_{\alpha}\right)^{-k / 2}
$$

relating to Sobolev spaces. Some commutation properties of the operators $\delta^{\alpha}$ with the operator of multiplication by $x^{\ell / 2}$ will be essential. We shall write $\delta^{\alpha} \frac{1}{x^{\ell / 2}}$ and $x^{\ell / 2} \delta^{\alpha}$ as shorthand for the action $\delta^{\alpha}\left(\frac{1}{(\cdot)^{\ell / 2}} f(\cdot)\right)(x)$ and $x^{\ell / 2} \delta^{\alpha}(f)(x)$. We state the following lemma whose proof (using (11)) is left to the reader.

Lemma 6. Let $\beta, \alpha>-1$, and $\ell \in \mathbb{N}$.

(i) $\delta^{\beta}=\delta^{\alpha}+\frac{\alpha-\beta}{2 \sqrt{x}}$.

(ii) $\delta^{\beta} \frac{1}{x^{\ell / 2}}=\frac{1}{x^{\ell / 2}} \delta^{\beta+\ell}$.

(iii) If $\beta>\ell-1$, then $\frac{1}{x^{\ell / 2}} \delta^{\beta+\ell}=\frac{1}{x^{\ell / 2}} \delta^{\beta-\ell}-\frac{\ell}{x^{(\ell+1) / 2}}$.

(iv) If $\beta>\ell-1$, then $\delta^{\beta} x^{\ell / 2}=x^{\ell / 2} \delta^{\beta-\ell}$.

(v) $\left(\delta^{\beta}\right)^{*}=-\delta^{\alpha}+\frac{1}{2}\left(\sqrt{x}-\frac{\alpha}{\sqrt{x}}\right)+\frac{1}{2}\left(\sqrt{x}-\frac{\beta+1}{\sqrt{x}}\right)$. 
Lemma 7. Let $\alpha>-1$ and $k \in \mathbb{N}$. Then

$$
\left(\delta^{\alpha}\right)^{k}=\sum_{0 \leq p \leq m+1, p+m=k-1} \frac{c_{m}}{x^{p / 2}} \delta^{\alpha+m} \circ \cdots \circ \delta^{\alpha} .
$$

Proof. Let $p<m+1$. By using Lemma 6 we have

$$
\begin{aligned}
\delta^{\alpha} & \left(\frac{1}{x^{p / 2}} \delta^{\alpha+m} \circ \cdots \circ \delta^{\alpha}\right)=\frac{1}{x^{p / 2}} \delta^{\alpha+p} \circ \delta^{\alpha+m} \circ \cdots \circ \delta^{\alpha} \\
\quad= & \frac{1}{x^{p / 2}}\left(\delta^{\alpha+m+1}+\frac{m+1-p}{2 \sqrt{x}}\right) \circ \delta^{\alpha+m} \circ \cdots \circ \delta^{\alpha} \\
\quad= & \frac{1}{x^{p / 2}} \delta^{\alpha+m+1} \circ \delta^{\alpha+m} \circ \cdots \circ \delta^{\alpha}+\frac{1}{x^{p / 2}} \frac{m+1-p}{2 \sqrt{x}} \delta^{\alpha+m} \circ \cdots \circ \delta^{\alpha} .
\end{aligned}
$$

If $p=m+1$ we have

$$
\delta^{\alpha}\left(\frac{1}{x^{p / 2}} \delta^{\alpha+m} \circ \cdots \circ \delta^{\alpha}\right)=\frac{1}{x^{p / 2}} \delta^{\alpha+m+1} \circ \delta^{\alpha+m} \circ \cdots \circ \delta^{\alpha} .
$$

Then

$$
\begin{aligned}
& \left(\delta^{\alpha}\right)^{k+1}=\delta^{\alpha}\left(\sum_{0 \leq p \leq m+1, p+m=k-1} \frac{c_{m}}{x^{p / 2}} \delta^{\alpha+m} \circ \cdots \circ \delta^{\alpha}\right) \\
& =\sum_{0 \leq p<m+1, p+m=k-1} \frac{c_{m}}{x^{p / 2}} \delta^{\alpha+m+1} \circ \delta^{\alpha+m} \circ \cdots \circ \delta^{\alpha} \\
& +\sum_{0 \leq p<m+1, p+m=k-1} \frac{c_{m}}{x^{p / 2}} \frac{m+1-p}{2 \sqrt{x}} \delta^{\alpha+m} \circ \cdots \circ \delta^{\alpha} \\
& +\sum_{0 \leq p=m+1, p+m=k-1} \frac{1}{x^{p / 2}} \delta^{\alpha+m+1} \circ \delta^{\alpha+m} \circ \cdots \circ \delta^{\alpha} \\
& =\sum_{0 \leq p<m, p+m=k} \frac{c_{m}}{x^{p / 2}} \delta^{\alpha+m} \circ \cdots \circ \delta^{\alpha} \\
& +\sum_{0 \leq q<m+1, q+m=k} \frac{c_{m}}{x^{q / 2}} \delta^{\alpha+m} \circ \cdots \circ \delta^{\alpha} \\
& +\sum_{0 \leq p=m, p+m=k} \frac{1}{x^{p / 2}} \delta^{\alpha+m} \circ \cdots \circ \delta^{\alpha} \\
& =\sum_{0 \leq p \leq m+1, p+m=k} \frac{c_{m}}{x^{p / 2}} \delta^{\alpha+m} \circ \cdots \circ \delta^{\alpha} .
\end{aligned}
$$

The standard induction argument completes the proof.

Lemma 8. Let $P_{m}(u, v)$ be a polynomial of degree $m$ in variables $u, v$, i.e.

$$
P_{m}(u, v)=a_{0} u^{m}+a_{1} u^{m-1} v+\cdots+a_{m} v^{m} .
$$


Assume that $\beta>m-1$. Then

$$
\delta^{\beta} P_{m}\left(\sqrt{x}, \frac{1}{\sqrt{x}}\right)=P_{m}^{1}\left(\sqrt{x}, \frac{1}{\sqrt{x}}\right) \delta^{\beta-m}+P_{m+1}^{2}\left(\sqrt{x}, \frac{1}{\sqrt{x}}\right)
$$

where $P_{m}^{1}$ and $P_{m+1}^{2}$ are polynomials of degrees $m$ and $m+1$.

Proof. Observe that

$$
\begin{aligned}
& P_{m}(\sqrt{x}, 1 / \sqrt{x}) \\
& \quad=a_{0} x^{m / 2}+a_{1} x^{(m-2) / 2}+\cdots+a_{m-1} x^{-(m-2) / 2}+a_{m} x^{-m / 2} .
\end{aligned}
$$

Let $0<\ell \leq m$. Then by using Lemma 6 we have

$$
\begin{aligned}
\delta^{\beta}\left(x^{\ell / 2}\right) & =x^{\ell / 2} \delta^{\beta-\ell}=x^{\ell / 2}\left(\delta^{\beta-m}+\frac{\ell-m}{2 \sqrt{x}}\right) \\
& =x^{\ell / 2} \delta^{\beta-m}+\frac{\ell-m}{2} x^{(\ell-1) / 2} .
\end{aligned}
$$

Let $\ell=-q<0$. Again by Lemma 6 we have

$$
\begin{aligned}
\delta^{\beta}\left(x^{\ell / 2}\right) & =\delta^{\beta} \frac{1}{x^{q / 2}}=\frac{1}{x^{q / 2}} \delta^{\beta-q}-\frac{q}{x^{(q+1) / 2}} \\
& =\frac{1}{x^{q / 2}} \delta^{\beta-m}+\frac{1}{x^{q / 2}}\left(\frac{q-m}{x^{1 / 2}}\right)-\frac{q}{x^{(q+1) / 2}} \\
& =\frac{1}{x^{q / 2}} \delta^{\beta-m}-\frac{m}{x^{(q+1) / 2}}=x^{\ell / 2} \delta^{\beta-m}-m x^{(\ell-1) / 2} .
\end{aligned}
$$

Lemma 9. Let $\ell, m$ be natural numbers such that $0<\ell \leq m$. For $\alpha>-1$ and $(\delta, p)$ satisfying $\left(\mathrm{C}_{\alpha}\right)$, the operators $\frac{1}{x^{\ell / 2}}\left(L_{\alpha+m}\right)^{-\ell / 2}$ and $x^{\ell / 2}\left(L_{\alpha+m}\right)^{-\ell / 2}$ are bounded on $L^{p}\left((0, \infty), y^{\delta} d y\right)$.

Proof. First, let $1=\ell=m$. We have already mentioned that the operator

$$
\left(\delta^{\alpha}\right)^{*}\left(L_{\alpha+1}\right)^{-1 / 2}=\left\{-\sqrt{x} \frac{d}{d x}+\frac{1}{2}\left(\sqrt{x}-\frac{\alpha+1}{\sqrt{x}}\right)\right\}\left(L_{\alpha+1}\right)^{-1 / 2}
$$

is bounded in $L^{p}\left((0, \infty), y^{\delta} d y\right)$ for $p, \delta$ satisfying $\left(\mathrm{C}_{\alpha}\right)$ (see (4)). Also the operator

$$
\left(\delta^{\alpha+1}\right)\left(L_{\alpha+1}\right)^{-1 / 2}=\left\{\sqrt{x} \frac{d}{d x}+\frac{1}{2}\left(\sqrt{x}-\frac{\alpha+1}{\sqrt{x}}\right)\right\}\left(L_{\alpha+1}\right)^{-1 / 2}
$$

is bounded in $L^{p}\left((0, \infty), y^{\delta} d y\right)$ for $p, \delta$ satisfying $\left(\mathrm{C}_{\alpha+1}\right)$. Hence both operators are bounded in $L^{p}\left((0, \infty), y^{\delta} d y\right)$ for $p, \delta$ satisfying $\left(\mathrm{C}_{\alpha}\right)$. Consequently, the operator $(\sqrt{x}-(\alpha+1) / \sqrt{x})\left(L_{\alpha+1}\right)^{-1 / 2}$ is also bounded. 
If $2(\alpha+1)<x$ then $0<\sqrt{x} \leq 2(\sqrt{x}-(\alpha+1) / \sqrt{x})$. We already know that $\left(L_{\beta}\right)^{-1 / 2}$ has positive kernel, hence for positive functions $f$ we have

$$
\begin{aligned}
\sqrt{x}\left(L_{\alpha+1}\right)^{-1 / 2} f(x) \leq & \sqrt{x}\left(L_{\alpha+1}\right)^{-1 / 2}(f)(x) \chi_{[0,2(\alpha+1)]}(x) \\
& +\left(\sqrt{x}-\frac{\alpha+1}{\sqrt{x}}\right)\left(L_{\alpha+1}\right)^{-1 / 2}(f)(x) \chi_{[2(\alpha+1), \infty)}(x) \\
\leq & \sqrt{2(\alpha+1)}\left(L_{\alpha+1}\right)^{-1 / 2}(f)(x) \chi_{[0,2(\alpha+1)]}(x) \\
& +\left(\sqrt{x}-\frac{\alpha+1}{\sqrt{x}}\right)\left(L_{\alpha+1}\right)^{-1 / 2}(f)(x) \chi_{[2(\alpha+1), \infty)}(x)
\end{aligned}
$$

The cases $\ell=1$ and $\ell<m$ can be proved as the previous one by using $\left(\delta^{\alpha+m-1}\right)^{*}\left(L_{\alpha+m}\right)^{-1 / 2}$ and $\delta^{\alpha+m}\left(L_{\alpha+m}\right)^{-1 / 2}$. Thus we obtain boundedness in $L^{p}\left((0, \infty), y^{\delta} d y\right)$ for $\delta, p$ satisfying $\left(\mathrm{C}_{\alpha+m-1}\right)$. By Remark 3 we obtain boundedness for $\delta, p$ satisfying $\left(\mathrm{C}_{\alpha}\right)$.

In the case $1<\ell \leq m$ we apply an induction argument. The operators

$$
R_{\alpha+m}^{\ell}=\left(\delta^{\alpha+m+\ell-1} \circ \cdots \circ \delta^{\alpha+m+1} \circ \delta^{\alpha+m}\right)\left(L_{\alpha+m}\right)^{\ell / 2}
$$

are bounded in $L^{p}\left((0, \infty), y^{\delta} d y\right)$ for $\delta, p$ satisfying $\left(\mathrm{C}_{\alpha+m}\right)$. On the other hand, the operators

$$
\widetilde{R}_{\alpha+m-\ell}^{\ell}=\left(\left(\delta^{\alpha+m-\ell}\right)^{*} \circ\left(\delta^{\alpha+m-\ell+1}\right)^{*} \circ \cdots \circ\left(\delta^{\alpha+m-1}\right)^{*}\right)\left(L_{\alpha+m}\right)^{-\ell / 2}
$$

are bounded in $L^{p}\left((0, \infty), y^{\delta} d y\right)$ for $\delta, p$ satisfying $\left(\mathrm{C}_{\alpha+m-\ell}\right)$ (see Theorem 8). In particular, both operators are bounded in $L^{p}\left((0, \infty), y^{\delta} d y\right)$ for $\delta, p$ satisfying $\left(\mathrm{C}_{\alpha}\right)$.

We observe that due to Lemma 6 we have, for $j=0, \ldots, \ell$,

$$
\begin{aligned}
\left(\delta^{\alpha+m-j}\right)^{*}= & -\delta^{\alpha+m+(j-1)} \\
& +\frac{1}{2}\left(\sqrt{x}-\frac{\alpha+m+(j-1)}{\sqrt{x}}\right)+\frac{1}{2}\left(\sqrt{x}-\frac{(\alpha+m-j)+1}{\sqrt{x}}\right) \\
= & -\delta^{\alpha+m+(j-1)}+\left(\sqrt{x}-\frac{\alpha+m}{\sqrt{x}}\right) .
\end{aligned}
$$

Therefore

$$
\begin{aligned}
\left(\delta^{\alpha+m-\ell}\right)^{*} \circ\left(\delta^{\alpha+m-\ell+1}\right)^{*} \circ \cdots \circ\left(\delta^{\alpha+m-1}\right)^{*} \\
=\left(-\delta^{\alpha+m+\ell-1}+\left(\sqrt{x}-\frac{\alpha+m}{\sqrt{x}}\right)\right) \circ\left(-\delta^{\alpha+m+\ell-2}+\left(\sqrt{x}-\frac{\alpha+m}{\sqrt{x}}\right)\right) \circ \cdots \\
\quad \cdots \circ\left(-\delta^{\alpha+m}+\left(\sqrt{x}-\frac{\alpha+m}{\sqrt{x}}\right)\right) \\
=\left(-\delta^{\alpha+m+\ell-1}+P_{1}(\sqrt{x}, 1 / \sqrt{x})\right) \circ\left(-\delta^{\alpha+m+\ell-2}+P_{1}(\sqrt{x}, 1 / \sqrt{x})\right) \circ \cdots \\
\quad \cdots \circ\left(-\delta^{\alpha+m}+P_{1}(\sqrt{x}, 1 / \sqrt{x})\right)
\end{aligned}
$$

where $P_{1}(\sqrt{x}, 1 / \sqrt{x})$ is the polynomial of first degree $\sqrt{x}-(\alpha+m) / \sqrt{x}$. 
Hence, by using Lemma 8 and an induction argument we get

$$
\begin{aligned}
\left(\delta^{\alpha+m-\ell}\right)^{*} \circ & \left(\delta^{\alpha+m-\ell+1}\right)^{*} \circ \cdots \circ\left(\delta^{\alpha+m-1}\right)^{*} \\
= & (-1)^{\ell} \delta^{\alpha+m+\ell-1} \circ \delta^{\alpha+m+\ell-2} \circ \cdots \circ \delta^{\alpha+m} \\
& +P_{1}(\sqrt{x}, 1 / \sqrt{x}) \delta^{\alpha+m+\ell-2} \circ \cdots \circ \delta^{\alpha+m} \\
& +P_{2}(\sqrt{x}, 1 / \sqrt{x}) \delta^{\alpha+m+\ell-3} \circ \cdots \circ \delta^{\alpha+m}+\cdots+P_{\ell}(\sqrt{x}, 1 / \sqrt{x}),
\end{aligned}
$$

where as usual $P_{j}(\sqrt{x}, 1 / \sqrt{x})$ denotes a polynomial of degree $j$. Now by using Lemma 5 we get

$$
\begin{aligned}
\widetilde{R}_{\alpha+m-\ell}^{\ell}= & (-1)^{\ell} R_{\alpha+m}^{\ell} \\
& +P_{1}(\sqrt{x}, 1 / \sqrt{x}) \circ\left(L_{\alpha+m+\ell-1}\right)^{-1 / 2} \circ T_{m, \alpha+m+\ell-1} \circ R_{\alpha+m}^{(\ell-1) / 2} \\
& +P_{\ell-1}(\sqrt{x}, 1 / \sqrt{x}) \circ\left(L_{\alpha+m+1}\right)^{-(\ell-1) / 2} \circ T_{m, \alpha+m+1} \circ R_{\alpha+m}^{1} \\
& +\cdots+P_{\ell}(\sqrt{x}, 1 / \sqrt{x}) \circ\left(L_{\alpha+m}\right)^{-\ell / 2},
\end{aligned}
$$

where $T_{m, \alpha+m+\ell-1}$ are multipliers analogous to those in Theorem 8. By Theorem 8 , Theorem 6 and the induction hypothesis on $\ell$, the operators $\widetilde{R}_{\alpha+m-\ell}^{\ell}, R_{\alpha+m}^{\ell}, P_{1}(\sqrt{x}, 1 / \sqrt{x}) \circ\left(L_{\alpha+m+\ell-1}\right)^{-1 / 2} \circ T_{m, \alpha+m+\ell-1} \circ R_{\alpha+m}^{(\ell-1) / 2}$ and $P_{\ell-1}(\sqrt{x}, 1 / \sqrt{x}) \circ\left(L_{\alpha+m+1}\right)^{-(\ell-1) / 2} \circ T_{m, \alpha+m+1} \circ R_{\alpha+m}^{1}$ are bounded for $\delta, p$ satisfying $\left(\mathrm{C}_{\alpha}\right)$, hence $P_{\ell}(\sqrt{x}, 1 / \sqrt{x}) \circ\left(L_{\alpha+m}\right)^{-\ell / 2}$ is bounded in the same range. Therefore by using the induction hypothesis on $\ell$ again, we see that an operator of the type $\left(a x^{\ell / 2}+b x^{-\ell / 2}\right) \circ\left(L_{\alpha+m}\right)^{-\ell / 2}$ is bounded. By an argument similar to the beginning of this proof we get the assertion.

TheOREM 10. Let $\alpha>-1$. Then the "Riesz" transforms $\left(\delta^{\alpha}\right)^{k}\left(L_{\alpha}\right)^{-k / 2}$ are bounded in $L^{p}\left((0, \infty), y^{\delta} d y\right)$ for $\delta, p$ satisfying $\left(\mathrm{C}_{\alpha}\right)$.

Proof. Let $0 \leq p \leq m+1$ and $p+m=k-1$. By Lemma 5 we have

$$
\left(\frac{1}{x^{p / 2}} \delta^{\alpha+m} \circ \cdots \circ \delta^{\alpha}\right)\left(L_{\alpha}\right)^{-k / 2}=\frac{1}{x^{p / 2}}\left(L_{\alpha+m+1}\right)^{-p / 2} T_{\mu} R_{\alpha}^{m+1},
$$

where $T_{\mu}$ is a multiplier defined on the system $\left\{\mathcal{L}_{n}^{\alpha+m+1}\right\}_{n \geq 0}$ which satisfies the hypothesis in Theorem 6 . Now Lemmas 9 and 7 and Theorem 8 give the result.

In order to analyze the possible coincidence for certain $\alpha, \delta$ and $p$ of the spaces $\mathcal{W}_{\alpha}^{k, p}\left(y^{\delta}\right)$ (see Definition 3 ) with the spaces considered in Section 3 we shall need the following reformulation of Lemma 9.

Lemma 10. Let $\ell, m$ be natural numbers such that $0<\ell \leq m+1$. For any $\alpha>0$ and $\delta, p$ satisfying $\left(\mathrm{C}_{\alpha-1}\right)$, the operators $\frac{1}{x^{\ell / 2}}\left(L_{\alpha+m}\right)^{-\ell / 2}$ are bounded on $L^{p}\left((0, \infty), y^{\delta} d y\right)$.

Proof of Theorem 2. By using Theorem 10 and the same arguments as at the beginning of the proof of Theorem 3, it is easy to prove (i). To see (ii), consider a function $f$ with support in $[0,1]$ such that $f(y)=y^{(\alpha+1) / 2}$ 
for $0<y<1 / 2, f(1)=0$ and $f$ is smooth in $[1 / 3,1]$. It is easy to see that $f, \delta^{\alpha} f$ and $\delta^{\alpha} \circ \delta^{\alpha} f$ belong to $L^{2}((0, \infty), d y)$. However, for $y \sim 0$, $\delta^{\alpha+1} \circ \delta^{\alpha} f \sim y^{(\alpha-1) / 2}$, that is, $\delta^{\alpha+1} \circ \delta^{\alpha} f$ is not in $L^{2}((0, \infty), d y)$.

Finally, let $f \in \mathcal{W}_{\alpha}^{2, p}\left(y^{\delta}\right)$. Then $\delta^{\alpha} f,\left(\delta^{\alpha}\right)^{2} f \in L^{p}\left((0, \infty), y^{\delta} d y\right)$, therefore (by Theorem 3) there exists $h \in L^{p}$ such that $\left(\delta^{\alpha}\right) f=\left(L_{\alpha}\right)^{-1 / 2} h$. Hence, by Lemma 6 ,

$$
\delta^{\alpha+1} \circ \delta^{\alpha} f=\delta^{\alpha} \circ \delta^{\alpha} f-\frac{1}{2 \sqrt{x}} \delta^{\alpha} f=\delta^{\alpha} \circ \delta^{\alpha} f-\frac{1}{2 \sqrt{x}}\left(L_{\alpha}\right)^{-1 / 2} h .
$$

Now Lemma 10 gives (iii).

5. Other Laguerre systems. We consider the orthonormal system in $L^{2}((0, \infty), d y)$ given by the Laguerre functions $\varphi_{k}^{\alpha}(y)=\mathcal{L}_{k}^{\alpha}\left(y^{2}\right)(2 y)^{1 / 2}$, $\alpha>-1$, where the $\mathcal{L}_{k}^{\alpha}$ are defined in (9). The functions $\varphi_{k}^{\alpha}$ are eigenfunctions of the operator

$$
\mathbf{L}_{\alpha}=\frac{1}{4}\left\{-\frac{d^{2}}{d y^{2}}+y^{2}+\frac{1}{y^{2}}\left(\alpha^{2}-\frac{1}{4}\right)\right\}
$$

In fact,

$$
\mathbf{L}_{\alpha}\left(\varphi_{k}^{\alpha}\right)=\left(k+\frac{\alpha+1}{2}\right) \varphi_{k}^{\alpha} .
$$

The operator $\mathbf{L}_{\alpha}$ can be "factorized" as

$$
\mathbf{L}_{\alpha}-\frac{\alpha+1}{2}=\left(\mathbf{D}_{\alpha}\right)^{*} \mathbf{D}_{\alpha}
$$

with

$\mathbf{D}^{\alpha}=\frac{1}{2}\left\{\frac{d}{d y}+y-\frac{1}{y}\left(\alpha+\frac{1}{2}\right)\right\} \quad$ and $\quad\left(\mathbf{D}^{\alpha}\right)^{*}=\frac{1}{2}\left\{-\frac{d}{d y}+y-\frac{1}{y}\left(\alpha+\frac{1}{2}\right)\right\}$, where $\left(\mathbf{D}^{\alpha}\right)^{*}$ is the formal adjoint of $\mathbf{D}^{\alpha}$ with respect to the Lebesgue measure. Then

$$
\mathbf{D}^{\alpha}\left(\varphi_{k}^{\alpha}\right)=-\sqrt{k} \varphi_{k-1}^{\alpha+1} \quad \text { and } \quad\left(\mathbf{D}^{\beta-1}\right)^{*}\left(\varphi_{k}^{\beta}\right)=-\sqrt{k+1} \varphi_{k+1}^{\beta-1} .
$$

As in Sections 3 and 4, the Riesz transforms can be defined as $\mathbf{R}_{\alpha}^{k}=\mathbf{D}^{\alpha+k-1} \circ \cdots \circ \mathbf{D}^{\alpha}\left(\mathbf{L}_{\alpha}\right)^{-k / 2}, \quad$ alternatively $\left(\mathbf{D}^{\alpha}\right)^{k}\left(\mathbf{L}_{\alpha}\right)^{-k / 2}, \alpha>-1$.

Let $V$ be the operator defined by $V f(y)=(2 y)^{1 / 2} f\left(y^{2}\right)$. Let $2 \delta=\gamma+$ $p / 2-1$. Then $\|V f\|_{L^{p}\left((0, \infty), y^{\gamma} d y\right)}=2^{1 / 2-1 / p}\|f\|_{L^{p}\left((0, \infty), y^{\delta} d y\right)}$.

Proposition 1. Let $1<p<\infty$, and $\delta, \gamma$ be real numbers. Let $T$ be an operator defined over the set of finite linear combinations of Laguerre functions $\left\{\mathcal{L}_{k}^{\alpha}\right\}_{k}$. Then $T$ has a bounded extension from $L^{p}\left((0, \infty), y^{\delta} d y\right)$ into $L^{p}\left((0, \infty), y^{\delta} d y\right)$ if and only if $\mathbf{T}=V T V^{-1}$ has a bounded extension from $L^{p}\left((0, \infty), y^{\gamma} d y\right)$ into $L^{p}\left((0, \infty), y^{\gamma} d y\right)$, where $2 \delta=\gamma+p / 2-1$. 
An easy consequence of the above proposition and Theorems 8 and 10 is the following.

TheOREM 11. Let $\alpha>-1$ and let $f$ be a finite linear combination of Laguerre functions $\left\{\mathcal{L}_{k}^{\alpha}\right\}_{k}$. Then

(i) $e^{-t L_{\alpha}} f=V^{-1} e^{-t \mathbf{L}_{\alpha}} V f$,

(ii) $\left(L_{\alpha}\right)^{-s} f=V^{-1}\left(\mathbf{L}_{\alpha}\right)^{-s} V f$ for $s>0$,

(iii) $\delta^{\alpha} f=V^{-1} \mathbf{D}^{\alpha} V f$,

(iv) $R_{\alpha}^{k} f=V^{-1} \mathbf{R}_{\alpha}^{k} V f$.

Proposition 2. Let $\alpha>-1,1<p<\infty$, and $\gamma$ be a real number. Let $S$ be any one of the operators $\mathbf{L}^{-s}, s>0, \mathbf{R}_{\alpha}^{k},\left(\mathbf{D}^{\alpha}\right)^{k} \mathbf{L}^{-k / 2}, s>0$. Then $S$ has a bounded extension from $L^{p}\left((0, \infty), y^{\gamma} d y\right)$ into itself for $\gamma$ satisfying

$$
\left(\mathbf{C}_{\alpha}\right) \quad-1-\alpha p-p / 2<\gamma<\alpha p+3 p / 2-1 .
$$

Now in a parallel way to we did in Sections 3 and 4, we can define potential spaces and Sobolev spaces for the class of Laguerre functions $\left\{\varphi_{k}^{\alpha}\right\}_{k}, \alpha>-1$. Thus, given $\alpha>-1,1<p<\infty, s>0$ and $\gamma$ satisfying $\left(\mathbf{C}_{\alpha}\right)$ (see (16)), we define

$$
\mathfrak{U}_{\alpha, s}^{p}\left(y^{\gamma}\right)=\left(\mathbf{L}_{\alpha}\right)^{-s / 2}\left[L^{p}\left((0, \infty), y^{\gamma} d y\right)\right]
$$

with the norm $\|f\|_{\mathfrak{L}_{\alpha, s}^{p}\left(y^{\gamma}\right)}=\|g\|_{p, \gamma}$, where $\left(\mathbf{L}_{\alpha}\right)^{-s / 2} g=f$.

We denote by $U_{\alpha}^{k, p}\left(y^{\delta}\right)$ the set of functions $f$ in $L^{p}\left((0, \infty), y^{\gamma} d y\right)$ such that

$$
\mathbf{D}^{\alpha+m} \circ \cdots \circ \mathbf{D}^{\alpha+1} \circ \mathbf{D}^{\alpha} f \in L^{p}\left((0, \infty), y^{\gamma} d y\right), \quad 0 \leq m \leq k-1,
$$

with the norm

$$
\|f\|_{U_{\alpha}^{k, p}\left(y^{\gamma}\right)}=\|f\|_{p, \gamma}+\sum_{m=0}^{k-1}\left\|\mathbf{D}^{\alpha+m} \circ \cdots \circ \mathbf{D}^{\alpha+1} \circ \mathbf{D}^{\alpha} f\right\|_{p, \gamma} .
$$

Finally, let $\mathcal{U}_{\alpha}^{k, p}\left(y^{\delta}\right)$ denote the set of functions $f$ in $L^{p}\left((0, \infty), y^{\gamma} d y\right)$ such that $\left(\mathbf{D}^{\alpha}\right)^{m} f \in L^{p}\left((0, \infty), y^{\gamma} d y\right), 0 \leq m \leq k$, with the norm

$$
\|f\|_{\mathcal{U}_{\alpha}^{k, p}\left(y^{\gamma}\right)}=\sum_{m=0}^{k}\left\|\left(\mathbf{D}^{\alpha}\right)^{m} f\right\|_{p, \gamma}
$$

The following theorems are direct consequences of Theorems 3,2 and Propositions 2 and 11.

Theorem 12. Let $\alpha>-1,1<p<\infty, k \in \mathbb{N}$ and $\gamma \in \mathbb{R}$. Then:

(i) $U_{\alpha}^{k, p}\left(y^{\gamma}\right)=\mathfrak{U}_{\alpha, k}^{p}\left(y^{\gamma}\right)$, and the norms are equivalent.

(ii) Let $\gamma$ satisfy $\left(\mathbf{C}_{\alpha}\right)$. Then $\mathfrak{U}_{\alpha, k}^{p}\left(y^{\gamma}\right) \subset \mathcal{U}_{\alpha}^{k, p}\left(y^{\gamma}\right)$.

(iii) Let $-1<\alpha \leq 0$. Then $\mathfrak{U}_{\alpha, 2}^{2} \neq \mathcal{U}_{\alpha}^{2,2}$.

(iv) Let $\gamma$ satisfy $\left(\mathbf{C}_{\alpha-1}\right)$. Then $\mathfrak{U}_{\alpha, 2}^{p}\left(y^{\gamma}\right)=\mathcal{U}_{\alpha}^{2, p}\left(y^{\gamma}\right)$. 
Analogous results could be obtained for the systems of Laguerre functions $\ell_{k}^{\alpha}(y)=\mathcal{L}_{k}^{\alpha}(y) y^{-\alpha / 2}$ and $\psi_{k}^{\alpha}(y)=\sqrt{2} y^{-\alpha} \mathcal{L}_{k}^{\alpha}\left(y^{2}\right), \alpha>-1$. These systems are eigenfunctions of the differential operators

$$
\mathbb{L}_{\alpha}=-y \frac{d^{2}}{d y^{2}}-(\alpha+1) \frac{d}{d y}+\frac{y}{4}
$$

and

$$
\mathfrak{L}_{\alpha}=-\frac{1}{4}\left\{\frac{d^{2}}{d y^{2}}+\frac{2 \alpha+1}{y} \frac{d}{d y}-y^{2}\right\} .
$$

We leave to the interested reader the easy work, but boring unless the statements are needed for some application, of establishing a version of Theorem 12 for these systems.

6. Schrödinger equation. Consider the equation

$$
\left\{\begin{array}{l}
i \frac{\partial u(y, t)}{\partial t}=L_{\alpha} u(y, t), \\
u(y, 0)=f(y), \quad y \in(0, \infty), t \in \mathbb{R}
\end{array}\right.
$$

for some initial data $f$. Consider its solution

$$
u(y, t)=e^{i t L_{\alpha}} f(y)
$$

for $f$ in the space $L^{2}((0, \infty), d y)$. From a general result in [5] one can see that if $f \in \mathfrak{W}_{\alpha, s}^{2}$ with $s>1$, then $\lim _{t \rightarrow 0} e^{i t L_{\alpha}} f(y)=f(y)$ a.e. $y$. On the other hand, it is known (see [4] and [6]) that $\lim _{t \rightarrow 0} e^{i t \Delta} f(y)=f(y)$ for $f$ such that $\Delta^{1 / 8} f \in L^{2}$. We give the following intermediate result.

Theorem 13. If $f \in \mathfrak{W}_{\alpha, s}^{2}$ with $s>1 / 2$ then

$$
\lim _{t \rightarrow 0} e^{i t L_{\alpha}} f(y)=f(y)
$$

for almost every $y \in(0, \infty)$.

Proof. It is enough to prove that the maximal function

$$
T^{*} f=\sup _{t \in \mathbb{R}}\left|e^{i t L_{\alpha}} f\right|
$$

satisfies the inequality

$$
\int_{I} T^{*} f \leq C\|f\|_{\mathfrak{W}_{\alpha, s}^{2}}
$$

for all compact intervals $I$ of the real line not containing the origin, and $C$ a constant that may depend on the interval $I$ but not on $f$.

From [19, Theorem 8.91.2, p. 241] and (9), if $I$ is an interval that does not contain the origin, then there exist constants $C$ and $n_{0}$ such that

$$
\mathcal{L}_{n}^{\alpha}(x) \leq \frac{C}{n^{1 / 4}} \quad \text { for all } x \in I \text { and } n \geq n_{0} .
$$


Now, if $f$ belongs to $\mathfrak{W}_{\alpha, s}^{2}$ we can write

$$
f(y)=\sum_{n=0}^{\infty} a_{n} \mathcal{L}_{n}^{\alpha}(y)
$$

and thus

$$
\|f\|_{\mathfrak{W}_{\alpha, s}^{2}}=\left(\sum_{n=0}^{\infty}\left|a_{n}\right|^{2}\left(n+\frac{\alpha+1}{2}\right)^{s}\right)^{1 / 2} .
$$

By Tonelli's theorem, estimate (19) and Hölder's inequality, we get

$$
\begin{aligned}
\int_{I}\left|T^{*} f(y)\right| d y & \leq \int_{I} \sup _{t>0}\left|\sum_{n=0}^{\infty} a_{n} e^{i t(n+(\alpha+1) / 2)} \mathcal{L}_{n}(y)\right| d y \leq \sum_{n=0}^{\infty}\left|a_{n}\right| \int_{I}\left|\mathcal{L}_{n}^{\alpha}(y)\right| d y \\
& \leq C\left(C+\sum_{n=n_{0}}^{\infty} \frac{1}{n^{1 / 2}(n+(\alpha+1) / 2)^{s}}\right)^{1 / 2}\left(\sum_{n=0}^{\infty}\left|a_{n}\right|^{2}\left(n+\frac{\alpha+1}{2}\right)^{s}\right)^{1 / 2} \\
& \leq C\left(C+\sum_{n=n_{0}}^{\infty} \frac{1}{n^{1 / 2+s}}\right)^{1 / 2}\|f\|_{\mathfrak{W}_{\alpha, s}^{2}} .
\end{aligned}
$$

Since $s>1 / 2$, we have $1 / 2+s>1$ and the last series is convergent.

Acknowledgements. This research was partially supported by Ministerio de Educación y Ciencia (Spain), grants MTM2005-08350-C03-01 and PCI 2006-A7-0670, and grants from CONICET and ANPCyT (Argentina), PIP 5809 and PICT 25588.

\section{References}

[1] I. Abu-Falahah, R. Macias, C. Segovia and J. L. Torrea, Transferring strong boundedness among Laguerre orthogonal systems, preprint.

[2] J. J. Betancor, J. C. Fariña, L. Rodríguez-Mesa and A. Sanabria-García, Higher order Riesz transforms for Laguerre expansions, preprint.

[3] B. Bongioanni and J. L. Torrea, Sobolev spaces associated to the harmonic oscillator, Proc. Indian Acad. Sci. Math. Sci. 116 (2006), 337-360.

[4] L. Carleson, Some analytic problems related to statistical mechanics, in: Lecture Notes in Math. 779, Springer, 1980, 5-45.

[5] M. Cowling, Pointwise behaviour of solutions to Schrödinger equations, in: Lecture Notes in Math. 992, Springer, 1983, 83-91.

[6] B. Dahlberg and C. Kenig, A note on the almost everywhere behavior of solutions to the Schrödinger equation, in: Lecture Notes in Math. 908, Springer, 1982, 205-209.

[7] G. Garrigós, E. Harboure, T. Signes, J. L. Torrea and B. Viviani, A sharp weighted transplantation theorem for Laguerre function expansions, J. Funct. Anal. 244 (2007), 247-276.

[8] P. Graczyk, J. Loeb, I. A. López, A. Nowak and W. O. Urbina, Higher order Riesz transforms, fractional derivatives, and Sobolev spaces for Laguerre expansions, J. Math. Pures Appl. (9) 84 (2005), 375-405. 
[9] E. Harboure, C. Segovia, J. L. Torrea and B. Viviani, Power weighted $L^{p}$-inequalities for Laguerre-Riesz transforms, Ark. Mat. 46 (2008), 285-313.

[10] E. Harboure, J. L. Torrea and B. Viviani, Riesz transforms for Laguerre expansions, Indiana Univ. Math. J. 55 (2006), 999-1014.

[11] B. Muckenhoupt and D. W. Webb, Two-weight norm inequalities for the Cesàro means of Hermite expansions, Trans. Amer. Math. Soc. 354 (2002), 4525-4537.

[12] A. Nowak and K. Stempak, Riesz transforms and conjugacy for Laguerre function expansions of Hermite type, J. Funct. Anal. 244 (2007), 399-443.

[13] R. Radha and S. Thangavelu, Multipliers for Hermite and Laguerre-Sobolev spaces, J. Anal. 12 (2004), 183-191.

[14] - - - Holomorphic Sobolev spaces, Hermite and special Hermite semigroups and a Paley-Wiener theorem for the windowed Fourier transform, preprint.

[15] E. M. Stein, Singular Integrals and Differentiability Properties of Functions, Princeton Univ. Press, Princeton, 1970.

[16] -, Topics in Harmonic Analysis Related to the Littlewood-Paley Theory, Ann. of Math. Stud. 63, Princeton Univ. Press, Princeton, NJ, 1970.

[17] K. Stempak and J. L. Torrea, Poisson integrals and Riesz transforms for Hermite function expansions with weights, J. Funct. Anal. 202 (2003), 443-472.

[18] —, - Higher Riesz transforms and imaginary powers associated to the harmonic oscillator, Acta Math. Hungar. 111 (2006), 43-64.

[19] G. Szegö, Orthogonal Polynomials, Amer. Math. Soc. Colloq. Publ. 23, rev. ed., Providence, 1959.

[20] S. Thangavelu, Lectures on Hermite and Laguerre Expansions, Math. Notes 42, Princeton Univ. Press, Princeton, 1993.

[21] - On regularity of twisted spherical means and special Hermite expansions, Proc. Indian Acad. Sci. 103 (1993), 303-320.

Departamento de Matemática

Facultad de Ingeniería Qímica

Universidad Nacional del Litoral

and

Instituto de Matemática Aplicada del Litoral

Santa Fe 3000, Argentina

E-mail: bbongio@santafe-ceride.gov.ar
Departamento de Matemáticas Facultad de Ciencias Universidad Autónoma de Madrid 28049 Madrid, Spain

E-mail: joseluis.torrea@uam.es

Received April 30, 2008

Revised version October 15, 2008 